\title{
How might avatar appearance influence health-related outcomes? A systematic review and narrative meta-review
}

\author{
Oliver Clark ${ }^{1}$, Sarah Grogan ${ }^{1}$, and \& Jennifer Cole ${ }^{1}$ \\ ${ }^{1}$ Brooks Building \\ Department of Psychology \\ Faculty of Health Psychology and Social Change \\ Manchester Metropolitan University \\ M15 6GX \\ UK
}

\begin{abstract}
Research has suggested that positive health behaviour change may be achieved using representations of the self in virtual environments (avatars) to alter self-perception, and model behaviours. This systematic review aimed to assess studies exploring the role that avatar appearance plays in influencing health-related outcomes.

Journal databases, authored books and websites, and grey literature sources were searched for experimental studies in which avatar appearance was used to manipulate physical health-related outcomes in healthy populations. A total of 16 articles (17 studies, 39 effect sizes) addressing primary (e.g. exercise, diet, and smoking), and secondary (e.g. body satisfaction) health related attitudes and behaviours were included.

Eight studies reported significant main effects of avatar manipulation, which included avatar body type (varied by adiposity or muscularity), and Self Discrepancy Theory-based (SDT; e.g. actual, ideal, ought selves) customisation.

The review indicates a dearth of research on avatar appearance and healthrelated outcomes. The assignment of avatars with varying body types appeared to consistently affect immediate physical activity during exercise game play. The assignment of 'obese' avatars appeared to reduce positive behavioural outcomes; however the absence of control conditions make it unclear whether performance was improved in the 'thinner' condition, or reduced in the 'obese' condition. Results from this review indicate a need for a grounded, theory-driven approach to future avatar appearance-based studies.
\end{abstract}

Keywords: Avatars; health; computer game-play; systematic review 


\section{Introduction}

Videogames that aim to promote positive attitudes and behaviours are called serious games (Orji et al., 2013), and are often applied to health settings. For example, exercise (Matallaoui et al., 2017), distribution of medical information (Behm-Morawitz et al., 2016), and health-related negotiation and decision making training (Christensen et al., 2013) may all be gamified to some extent using videogames (McCallum, 2012). Invariably, serious videogames require a virtual environment for users to navigate, and therefore require a form of representation, or avatar, in this environment. Avatars have been succinctly defined by Nowak and Fox (2018) as follows:

an avatar is a digital representation of a human user that facilitates interaction with other users, entities, or the environment [p34].

An avatar is therefore distinct from agents which are controlled by a computer, and Doppelgangers which resemble a user, but do not facilitate interaction (Ahn et al., 2014). The mere appearance of an avatar alone has been shown to elicit behaviour change in virtual environments. For example, Yee et al. (2009) found that people controlling an "unattractive" avatar in a virtual environment disclosed less personal information, and maintained a larger interpersonal distance than those controlling an attractive avatar; this phenomenon is named the Proteus Effect. If physical appearance during a videogame (or other digitallymediated experiences) can alter attitudes and behaviours, then it has been suggested that digital self representation may have the potential to improve health-related outcomes. The current study aimed to review the literature on the impact of avatar appearance on healthrelated outcomes.

\section{Existing Reviews}

Two narrative review chapters (Ahn \& Fox, 2017a , 2017b), and two systematic reviews (Horne et al., 2019; Rheu et al., 2019) describe the literature on health and avatars. The narrative reviews offer interesting insights into the possible uses of avatars and agents (computer-controlled characters) in healthcare, and suggest that there are two unique affordances that avatars and agents have over traditional communication methods: interactivity (allowing users to have agency over the media content); and time acceleration (e.g. showing immediate weight loss and weight gain in response to health-related behaviours). However,

The authors declare that they had no conflicts of interest with respect to their authorship or the publication of this article. No funding was associated with the project The authors would like to acknowledge the help of Andrea Daly from Manchester Metropolitan University for her help in developing the search strategy. This pre-print was generated using PAPAJA (Aust \& Barth; 2017).

Correspondence concerning this article should be addressed to Oliver Clark, Brooks Building, Manchester Metropolitan University. E-mail: oliver.clark3@stu.mmu.ac.uk 
it is notable that none of the studies reviewed are related to avatars by the recent standard definition (Nowak \& Fox, 2018); and are better described as doppelgangers (i.e. object representations, rather than subject representations).

Horne et al. (2019) specifically addressed the use of avatars in a weight management context, and identified six studies that used avatar-based interventions. They conclude that avatars may have the potential to improve weight loss, but naturally more studies are needed to judge clinical efficacy [named the Oliver Twist argument by Valentine et al. (2010). Missing from this review is a discussion of the mechanisms behind avatar use, with avatars seemingly regarded as mere alternative mediators for more traditional interventions (e.g. education, motivational interviewing). However the attempts made to assess the quality of evidence using an established tool were beneficial (Sirriyeh et al., n.d.). The avatar studies reviewed in the Horne et al. (2019) article were arguably closer to efficacy studies for digitally-mediated interventions than studies investigating the unique contribution of avatars to health persuasion. Two studies created tailored or personalised avatars for participants, however one of these would not count as an avatar under the definition from Nowak and Fox (2018), since the model was a time-accelerated image of the participant under different weight conditions based on their current intake; this is more akin to appearance-related interventions such as facial morphing (e.g. Persson, Grogan, et al., 2018; Grogan et al., 2011).

Rheu et al. (2019) systematically reviewed health-related interventions using avatars, identifying 18 studies in which avatars were applied to health-related behaviours. The authors summarise the field, and highlight six characteristics that may be manipulated in avatar-based interventions, concluding that using an avatar that is physically active, fit, and similar looking to the participant is effective at promoting health-related behaviours. They also address potential risk of bias, using (an uncited version of) the Cochrane Tool for Assessing Risk of Bias (Higgins et al., 2011). The review by Rheu et al. (2019) is concise, wide in scope, and provides some interesting insights. However, we argue that the current the review uses more robust methodology, is more focused in its aims, covers a broader range of outcomes, and considers the effect sizes and confidence intervals generated by the studies.

There are also some potential shortcomings in the Rheu et al. (2019) paper. Their definition of an avatar included doppelgangers, which is perhaps too broad. Moreover, their definition of health behaviour was limited to diet and exercise, which in turn was perhaps too narrow. Further, the authors conflate the two key theories of digital behaviour change, the Proteus Effect (Yee et al., 2009) and Virtual Self Modelling (Fox \& Bailenson, 2009); these have very different theoretical roots and implications.

Neither systematic review considered effect size, with the weight of evidence being determined by significant $p$-values. This is problematic for a variety of reasons. $P$ values may be artificially inflated at low sample sizes, and when sample sizes are overly large, negligible effects may be observed as significant. Moreover, the effects of interest in some studies may not have been tested using significance testing. For instance, a $2 \mathrm{X} 2$ factorial design including avatar appearance and gaming task as factors may only be presented as a single significant $\mathrm{F}$ value, but actually contains two treatments (avatar and task), one of 
which might have a small effect size, and the other a large effect size, meaning that the confidence in the effect of avatars is inflated, when it is in fact the task that is driving the difference.

\section{Current Review}

A pre-registered, transparent approach was adopted, and we followed preferred reporting guidelines (PRISMA, Moher et al., 2015). We also consulted a wider range of sources than Rheu et al. (2019) and Horne et al. (2019), including grey literature searches by contacting authors and searching thesis repositories. We queried more academic databases, ran hereditary and ancestry searches, and used a continuous searching method to identify papers that were published after the initial searches were conducted.

Since visual avatars are very common, and as such their appearance is the most salient feature, this review specifically addresses avatar appearance. The definition of health-related behaviours is wider, including smoking, UV protection, and safe sexual practices as outcomes in the literature search. A wider outcome net allows inferences to be made about hetereogeneity of effects across dependent variables, and the generalisability of the approach. Since body satisfaction and health-related behaviours are broadly related (Grogan, 2017), and research suggests that exposure to idealised media may have negative consequences in some circumstances (e.g. Wasilenko et al., 2007), body image was included as a secondary outcome in the current review.

Finally, we base our conclusions on effect size estimates and confidence intervals and deconstruct factorial designs to isolate the contribution of avatar appearance on health outcomes.

\section{Objectives}

There were three main objectives of this review:

1. To determine the efficacy of avatar-appearance in improving health-related behaviour outcomes including direct observable behaviours, and direct (e.g. intentions), and indirect (e.g. related to body dissatisfaction) self reported measures.

2. To address the quality of the methodology within the studies using existing tools (e.g. the Cochrane tool for assessing bias, Statcheck, etc).

3. To use meta-analytical techniques to estimate the overall effect size for avatar healthrelated persuasion.

\section{Method}

The guidelines from the PRISMA statement were adhered to in the undertaking of this systematic review (Moher et al., 2015). The following is an abbreviated method section $^{1}$.

\footnotetext{
${ }^{1}$ Full details can be found in the digital appendix https://osf.io/k7jrn/.
} 


\section{Search strategy}

The studies for this review were located using electronic database searches of Medline, PsyArticles, PsychInfo, Pubmed, Web of Science, and Web of Knowledge. Unpublished papers were sought using the electronic thesis repository Proquest, and by contacting prominent authors in the field. The search strategy was developed with the assistance of an academic librarian and the terms included avatar, digital self, videogame, virtual reality, health, exercise, behaviour, change, attitude, or intention ${ }^{2}$. Searches were not restricted to English journals, although only languages that could be translated using Google Translate were considered. Reference lists, chapters, and author websites were also consulted.

\section{Continuous Searching}

Alerts were set for NCBI and Scopus. An email was received by the author whenever a study meeting the criteria of the search terms was released. Each paper was checked using the same three point method as described below (title, abstract, full text screening).

\section{Eligibility Criteria}

\section{Types of Studies.}

All quantitative studies looking at effects of avatar appearance on health-related outcomes, using either comparative or within-group methods were considered. This included conference papers, peer-reviewed articles, and unpublished dissertations.

\section{Types of participants.}

Only studies investigating non-clinical populations were eligible for inclusion. Studies on children and adults were included.

\section{Types of intervention.}

Only studies manipulating some element of avatar appearance were included in the review. Participants also had to have some control over the avatar. As such, studies in which the only manipulation was customisation (e.g. Waddell et al., 2015), and those that used avatars but did not manipulate the appearance between (or within) conditions (e.g. Morgan et al., 2014) were excluded. Manipulations included variation induced through instructions given to participants during customisation, and studies in which different avatars were assigned to a participant. When reviewing studies for inclusion, the definition of 'avatar' was identified as a major source of variation. The research question was consulted again, and the research team decided to define an avatar as a representation of the self in a virtual environment. Excluded from this definition were studies on virtual self modelling, which rely on the participant seeing the model as different from themselves, or as a 'doppelgänger'. In these studies, participants typically observe the model, and have no control or influence over it. This was fundamentally different from the majority of studies selected for inclusion. VSM studies may warrant a separate systematic review. Similarly, virtual pet studies were

\footnotetext{
${ }^{2}$ Full search terms with boolean operators may be found in the appendix.
} 
also excluded on a similar basis, in that the avatar was not intended to represent the participant, but to act as a model that reflected their behavioural decisions such as eating breakfast (Ahn et al., 2015).

\section{Types of outcome measures.}

Primary outcome measures included behaviours such as exercise and dieting, and observed and self reported changes in health-related attitudes, beliefs, and intentions. Secondary outcome measures pertaining to body image and body dissatisfaction were also of interest.

\section{Study selection}

Studies from all sources were aggregated into a master RIS (Research Information Systems) file and screened for title and abstract using Rayyan (Elmagarmid et al., 2014; Khabsa et al., 2015). Initial screening was conducted by the author. The second and third authors blind-reviewed $10 \%$ of the judgements made by the author. Conflicts were discussed at research meetings and were attributed to misunderstandings of the operational definitions, and were subsequently resolved. The studies that were included after initial screening were subjected to full-text screening. Eligibility of ambiguous papers was discussed and resolved at research meetings.

\section{Data Extraction}

Data were extracted following the method of a previous literature review (Freijy \& Kothe, 2013), and involved extracting the country, health outcome, sample characteristics, comparison group manipulations and relevant results. Inferential statistics for the relevant results were also extracted. Unless otherwise justified in a pre-registration, the highest acceptable error rate to represent a meaningful effect for this review was 0.05 , and so any studies reporting 'marginal', or 'trends towards significance' were interpreted as not significant. In addition to p-values, Cohen's D Effect sizes and variances were calculated, either from reported means and standard deviations (where available), or through transformation using degrees of freedom and $F$ statistics using the fes() function from the compute.es package in R (Del Re, 2013). Where factorial designs were adopted, marginal effect sizes isolating the avatar appearance-related treatment were calculated by hand (formulae can be found in the supplemental data). "No avatar" or "no play" control conditions were excluded as effects sizes [8,9], as were cases in which the task varied but not the avatar. For example, in study [10], participants either had a customised or assigned avatar during a jogging game, or a customised avatar during a bowling game. The latter was excluded. Since these designs were not anticipated, the decision to exclude individual effects was made during the review process. The process for this can be found in the supplementary materials. In four cases $[0,8,9,12]$, means and standard deviations were obtained from information provided from corresponding authors. 


\section{Assessment of Risk of Bias}

Risk of bias was assessed using the Cochrane Collaboration tool (Higgins et al., 2011). Each study was assessed for selection bias (randomised assignment of participants, and concealment of allocation from personnel), performance bias (blinding participants to the intervention), detection bias (blinding of assessors and other personnel, attrition bias (loss of participants), and reporting bias (selective reporting of outcomes). One study [0] was conducted by the research team, and so this study was reviewed for risk of bias by a third party. In Figure 4, effect sizes were categorised by risk of bias by coding each + in Table 7 as coded as 1 , each . as 0 , and each - as -1 and then summing the codes. Any study that scored below 0 was categorised as unknown, with positive and negative scores indicating high and low bias respectively.

\section{Data Synthesis}

Due to the small number of studies, and degree of heterogeneity in outcomes and methods, a full meta-analysis is not appropriate for making inferences about the literature. Although a fair number of effect sizes is presented (39), these are firstly not independent and should not be synthesised outside of a multi-level model (this is not being conducted due to sample size and heterogeneity); secondly an aggregated effect size of intentions, attitudes, beliefs, body satisfaction, and behaviour is less a case of comparing apples and oranges, with the amount of heterogeneity between studies making such an evaluation more akin to comparing the individual items of a high-end pre-packaged fruit salad. As can be seen in Figures ?? and 3, once this fair number has been divided into similar categories, there are very few effects per cell. Instead of the planned multi-level meta-regression that was pre-registered, a qualitative, narrative approach to the synthesis of the data was adopted in which effect sizes and confidence intervals of similar studies are be presented together, and the variation in these are discussed in terms of risk of bias (Valentine et al., 2010). Random effect estimates will be presented for the subgroups using the rma() function from the metafor package (Viechtbauer, 2010), since despite the small number of studies, this information may still be useful (Valentine et al., 2010), however these must not be interpreted as meta-analytic effect size since the effects from which they are calculated are not independent. Multiple effect sizes are presented from each study, meaning that the effect sizes will be correlated within studies, and the aggregated estimate will be a vastly inaccurate estimate of the population effect.

\section{Results}

Searches yielded 17 studies from 16 articles to be included in the review. Figure 1 presents the PRISMA flow diagram for study selection ${ }^{3}$. In the results and discussion below, the included studies are referenced using the identification codes from Table 2 in square parentheses.

\footnotetext{
${ }^{3}$ Full details about the yields from each source can be found in the supplemental materials.
} 


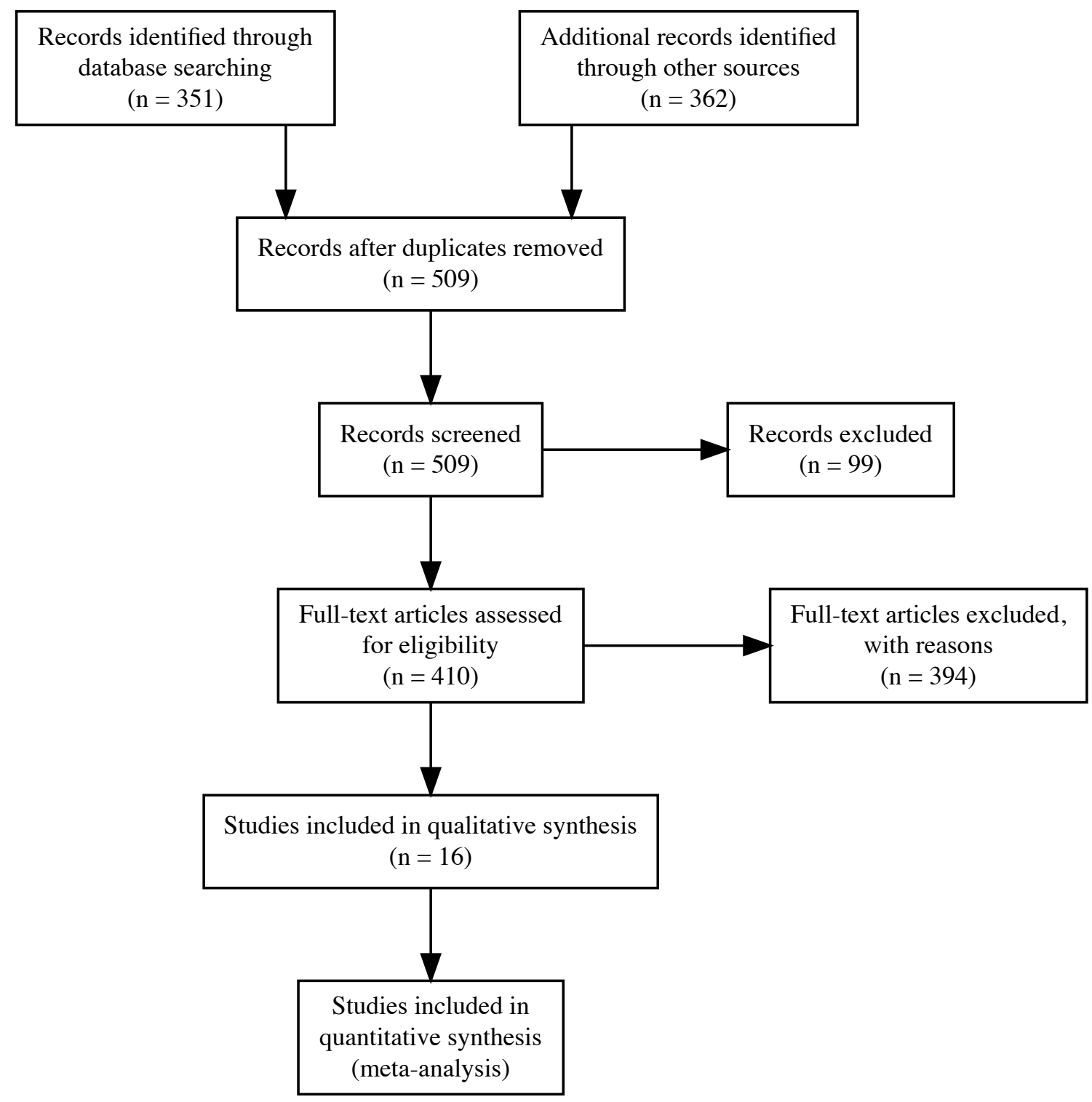

Figure 1

Prisma Flow chart 


\section{Study Categorisation}

The clearest difference in studies was whether participants customised, or were assigned an avatar. The majority of studies had an assigned avatar condition $[\mathrm{N}=11]$, although some studies also included customisation as a factor in the design matrix $[1,5]$. A second factor was the type of manipulation employed, of which there were four:

1. Ideal: Participants were assigned, or customised and avatar that reflected either their Ideal, Actual, or Ought selves, in line with the self models and concepts from SelfDiscrepancy Theory (Higgins, 1987)

2. Idealised: Partcipants were assigned an avatar that reflected idealised media norms such as muscularity or extreme thinness.

3. Obese: Participants were assigned to either "slim" or "obese" avatar conditions.

4. Self: Participants were assigned an avatar that resembled themselves, or a stranger.

Although this categorisation was not pre-registered, effect sizes will be clustered by these distinctive designs for ease of interpretation.

\section{Study Characteristics}

Table 2 provides the study characteristics and identification numbers for the dataset. All but one of the studies were peer reviewed journal articles published in a range of journals including: 10 from human computer interaction journals $[1,5,7,8,9,11,12,14,15,16]$; four from psychology $[2,4,6,12]$; one from a health and environment journal [10]; and one from a games and health journal [3]. One study had previously contributed towards a doctoral thesis [5], and one study was unpublished at the time of the review [0].

Primary target health-related behaviours included smoking [13], exercise/physical activity $[0,3,4,7,10,11,12]$, diet $[2,3,6,13,16]$, "maintaining a general healthy lifestyle" [5], and Human Papillimamilliary Virus vaccination [1].

Regarding the populations that were sampled from, one study was conducted only with overweight children from Singapore [7], and one study was conducted only with overweight Spanish women [10]. The remainder of the studies were sampled mostly from undergraduate populations, with four studies selecting only males $[1,9,12,15]$, and three selecting only females $[8,10,12]$. No studies reported sample size calculations based on statistical power analysis, although one reported an a posteriori power analysis [2]. 
Table 1

\begin{tabular}{llll}
\hline ID & \multicolumn{1}{c}{ Reference, country, and study design } & \multicolumn{2}{c}{ Health-related outcome } \\
\hline 0 & Clark et al (2019); UK; Randomised Trial & Physical activity & $\mathrm{N}=54$ \\
1 & Darville et al (2018) USA Randomised trial & HPV vaccination & $\mathrm{N}=16$ \\
2 & Jin (2012) USA Randomised trial & Diet & $\mathrm{N}=15$ \\
3 & Joo and Kim (2017) Korea Randomised trial & Exercise and eating & $\mathrm{N}=12$ \\
4 & Kastenmüller et al (2013) UK & Physical activity & $\mathrm{N}=42$ \\
5 & Kim and Sundar (2012) USA Randomised Trial & Healthy Lifestyle & $\mathrm{N}=95$ \\
6 & Kuo et al (2016) Taiwan Randomised Trial & Diet & $\mathrm{N}=76$ \\
7 & Li et al (2013) Singapore Factorial Randomised trial & Exercise & $\mathrm{N}=14$ \\
8 & Matthews et al (2016a) USA Randomised trial & Body satisfaction & $\mathrm{N}=14$ \\
9 & Matthews et al (2016b) USA Randomised trial & Body satisfaction & $\mathrm{N}=19$ \\
10 & Navarro et al (2020) Spain Randomised Trial & Physical Activity Self efficacy Anxiety & $\mathrm{N}=42$ \\
11 & Peña and Kim (2014) USA Randomised trial & Exercise & $\mathrm{N}=96$ \\
12 & Peña et al (2016) USA Randomised trial & Exercise & $\mathrm{N}=96$ \\
13 & Sah et al (2016) USA Randomised trial & Eating & $\mathrm{N}=13$ \\
14 & Song et al (2013) USA Randomised trial & Smoking & $\mathrm{N}=62$ \\
15 & Sylvia et al (2014) USA Randomised trial & Body satisfaction & $\mathrm{N}=51$ \\
16 & Velhurst et al (2018) Japan Randomised trial & Health food shopping & $\mathrm{N}=23$ \\
17 & Waddell et al (2015) USA Randomised trial & Exercise and nutrition & $\mathrm{N}=13$ \\
\hline
\end{tabular}




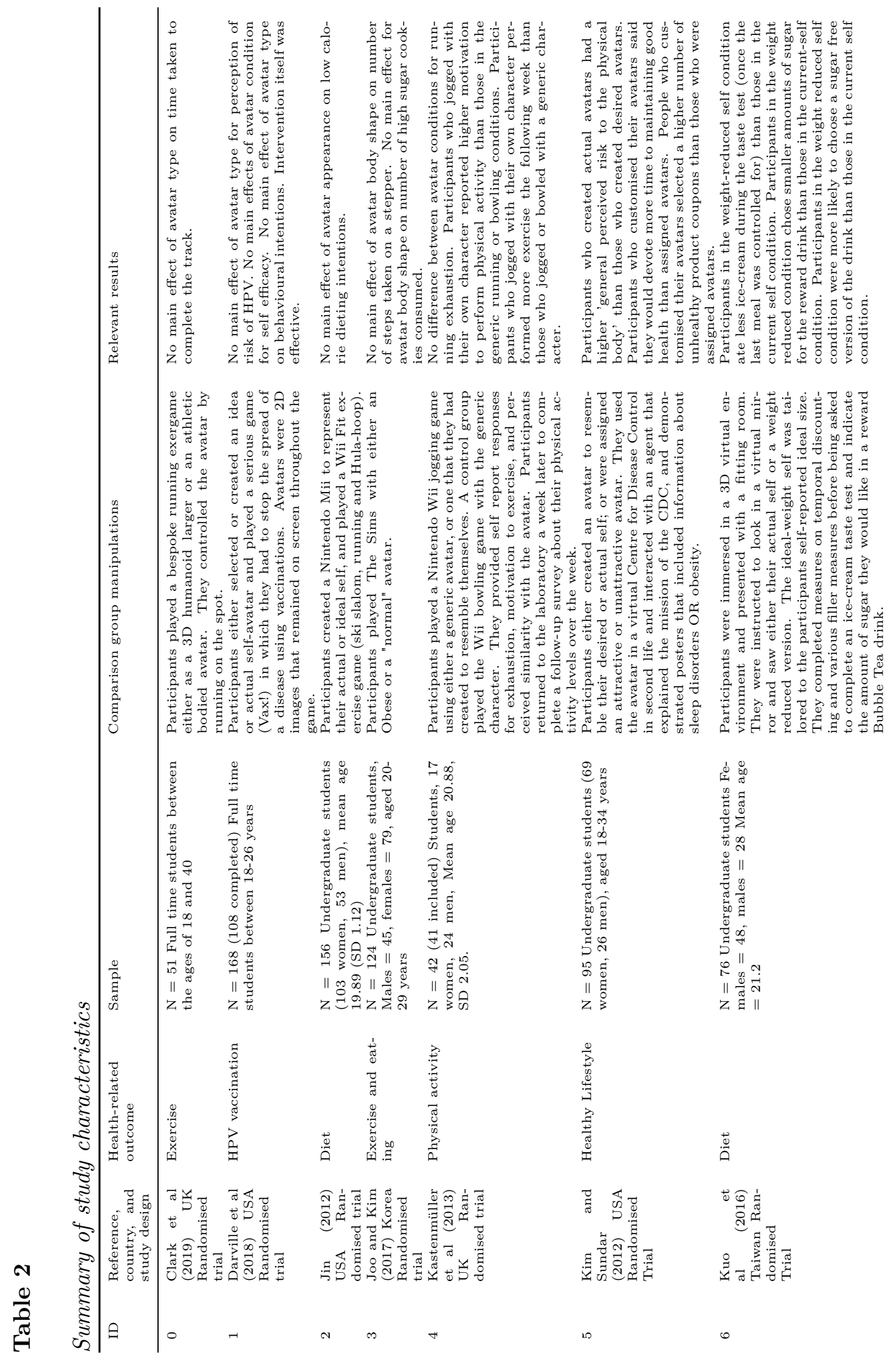



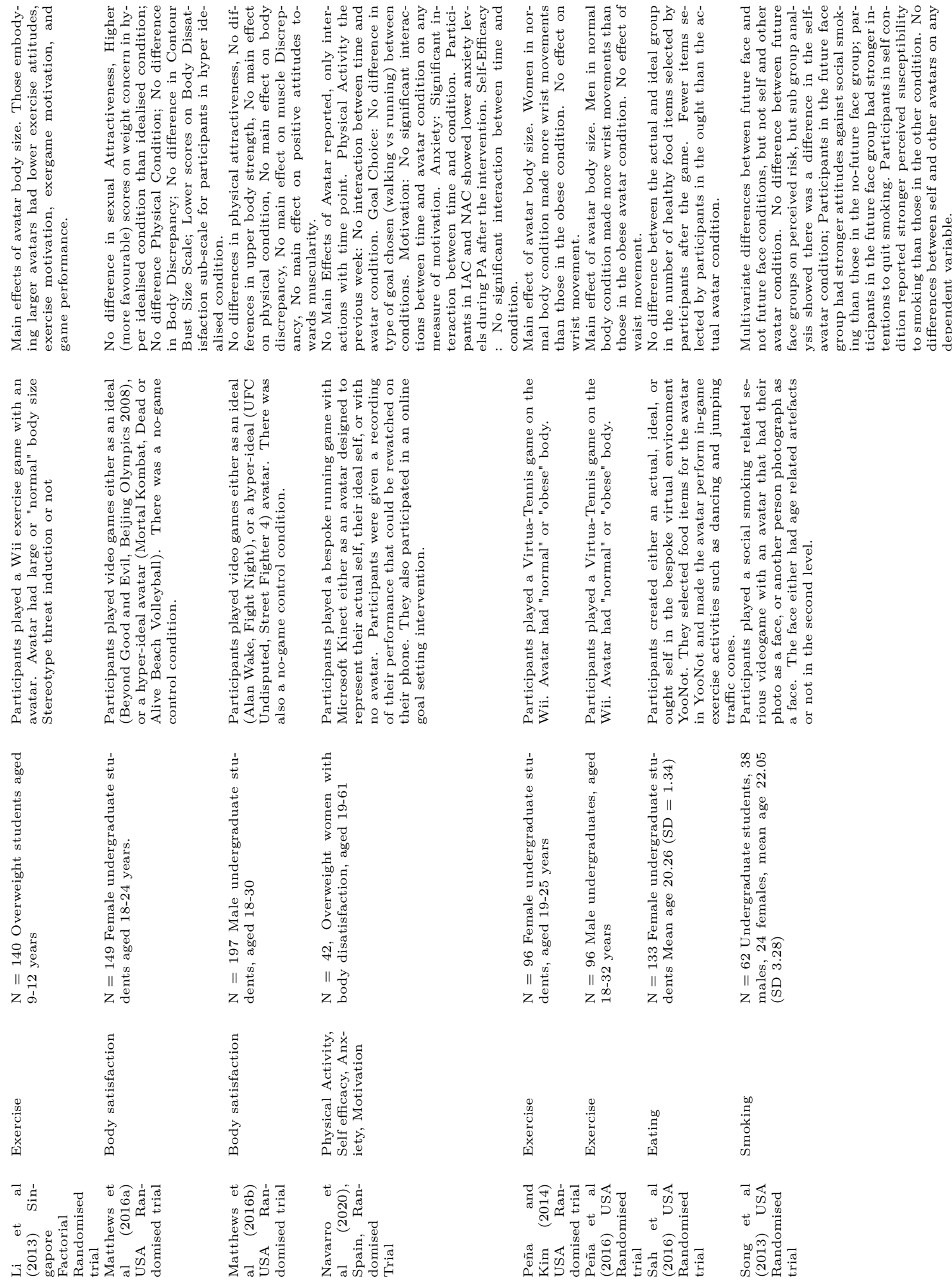


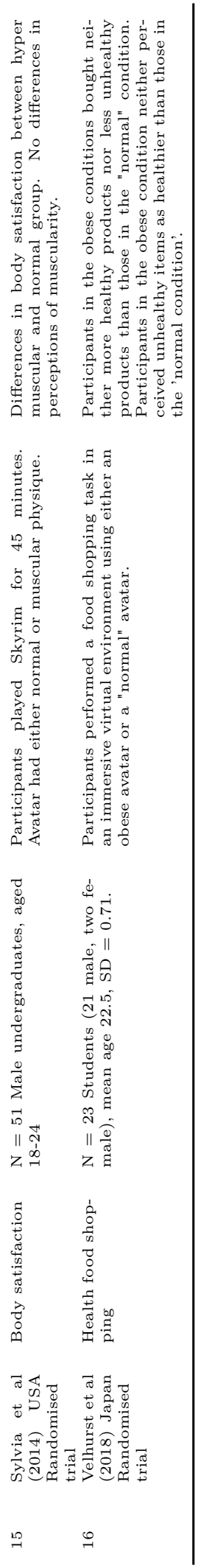




\section{Reliability and Validity}

For self-report measures, all but two studies $[6,16]$ reported a measure of internal consistency (Cronbach's alpha, $\alpha$ ). No study provided a justification for selecting $\alpha$ rather than other measures of internal consistency (e.g. Omega, $\omega$ ). Internal reliability of measurement ranged from 0.7 to 0.94 . Only one study discussed the validity of its measures [10]. In some cases, standardised measures were used, and such information could, in principle, be acquired by following references. Table 4 provides details of the validity and reliability measures discussed in the papers. 
Table 3

\begin{tabular}{|c|c|c|c|c|c|c|}
\hline Health-related outcome & ID & Behaviour & Attitude & Intention & Manipulation & Michie Tax \\
\hline Physical activity & 0 & NS & . &. & OB & NA \\
\hline HPV vaccination & 1 & . & NS & NS & SDT & 13.1 \\
\hline Diet & 2 & . & . & NS & SDT & 13.1 \\
\hline Exercise and eating & 3 & NS & . & . & OB & $6.1 ; 6.2$ \\
\hline Physical activity & 4 & + & + & . & CUST & 6.2 \\
\hline Healthy Lifestyle & 5 & . & + & \pm & SDT & 13.1 \\
\hline Diet & 6 & + & . & - & ID & $13.1 ; 16.3$ \\
\hline Exercise & 7 & + & + & + & OB & $6.2 ; 13.5$ \\
\hline Body satisfaction & 8 & . & \pm & . & ID & 6.2 \\
\hline Body satisfaction & 9 & . & NS & . & ID & 6.2 \\
\hline Physical Activity Self efficacy Anxiety & 10 & NS & NA & NS & SDT & NA \\
\hline Exercise & 11 & + & . & . & $\mathrm{OB}$ & $6.2 ; 13.5$ \\
\hline Exercise & 12 & + & . & . & $\mathrm{OB}$ & $6.2 ; 13.5$ \\
\hline Eating & 13 & - & . & . & SDT & 13.1 \\
\hline Smoking & 14 & . & + & + & SELF & $16.3 ; 9.3$ \\
\hline Body satisfaction & 15 & . & - & . & ID & 6.2 \\
\hline Health food shopping & 16 & NS & NS & . & $\mathrm{OB}$ & 13.5 \\
\hline Exercise and nutrition & 17 & NS & . & NS & SOT & NA \\
\hline
\end{tabular}




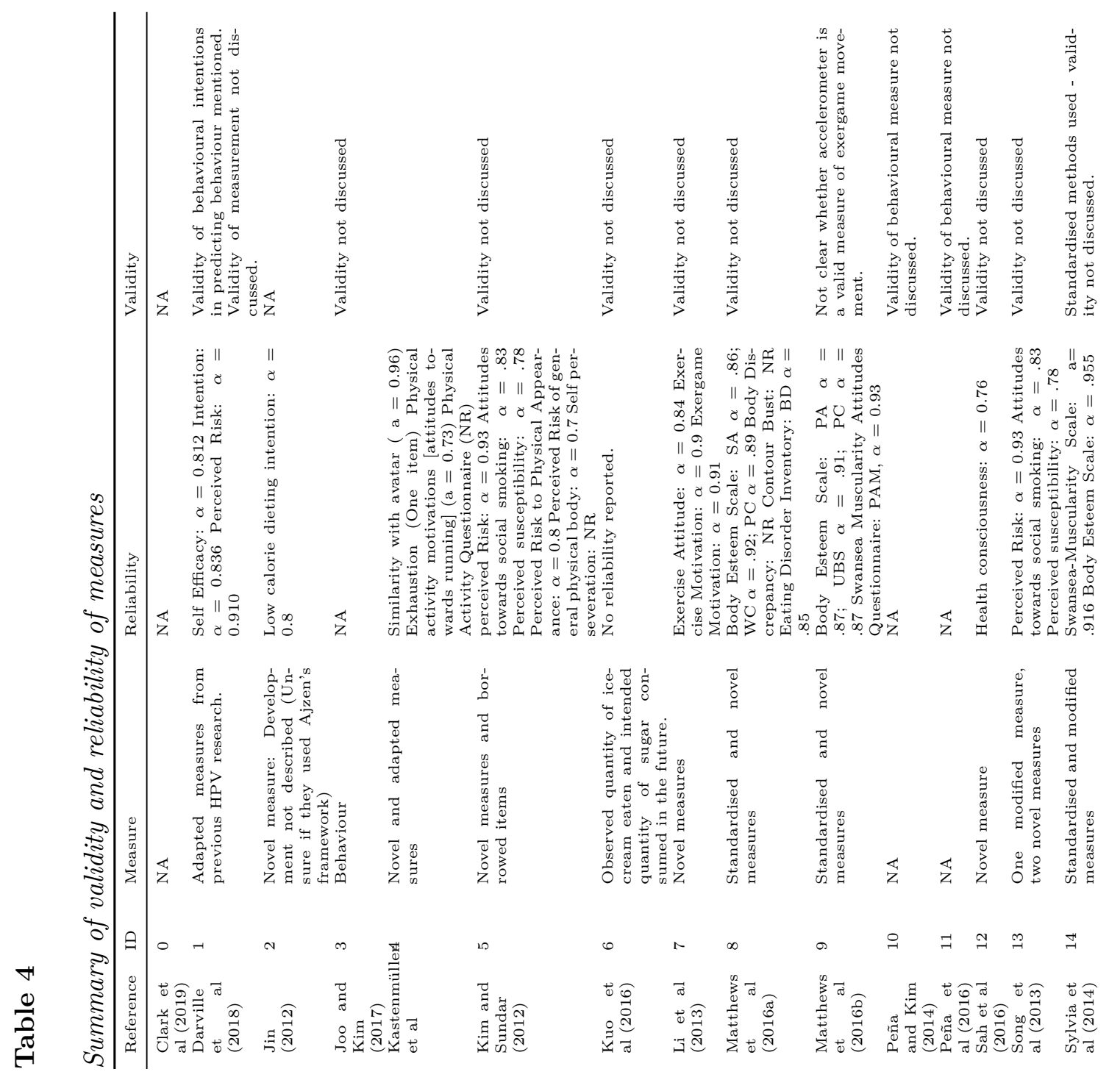




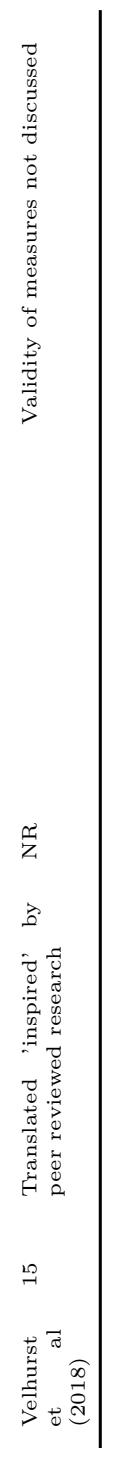




\section{Experimental Paradigms}

Although there was a visual appearance element to the avatar manipulations in all of the studies, the task, type of variation, and type of avatar varied considerably both between and within studies. A between subject design was employed in studies where avatars varied by body size $[0,3,6,7,11,12,16]$, and muscularity $[8,9,15]$. Within subject factors were employed in two studies in which change in outcomes over time was of interest $[1,10]$, but avatar treatments were always between subject factors.

In general, the studies in which participants customised their avatars were more complex, with both assignment and customisation being used as manipulations in some cases [e.g. 1, 5], and only customisation used in others [e.g. 10]. Moreover, the customisation paradigms were far more varied between studies. There were 10 separate manipulations, including different combinations of the self-models and self concepts described by Self-Discrepancy Theory (Higgins, 1987). These were used as instructions during avatar customisation (e.g. "create an avatar that reflects your ideal self"). Participants were instructed to create an avatar that reflected their actual, ideal, or ought selves in one study [13], and actual or ideal selves in four studies $[1,2,5,10]$. For the purposes of synthesis, these have all been categorised as 'ideal self' paradigms. .

\section{Evidence for effectiveness}

Figures 2 and 3 provide forest plots of the effect sizes calculated and confidence intervals from the results sections of the papers ${ }^{4}$. Figure ?? presents the effect sizes and confidence intervals for assigned and customised avatar studies separately, whereas Figure 3 presents each outcome (Behaviour, Intentions, Attitudes) separately, and categorised by treatment. Regarding statistical significance, seven of the assigned and two of the customised avatar effect size confidence intervals did not include zero.

The majority of studies $[\mathrm{N}=13]$ reported direct effects (rather than/in addition to interactions) of avatar appearance on health related attitudes and behaviours, with eight reporting positive changes (an increase in health related attitudes, beliefs, intentions or behaviours) $[4,5,6,7,11,12,13,14]$, and five reporting negative effects $[6,8,14,15]$. For manipulations involving obese avatars (i.e. an assumed 'non-ideal', or 'feared' self avatar), performance during an exergame was reduced in three studies $[7,11,12]$, whereas manipulations involving idealised avatars (i.e. an assumed ideal avatar) sometimes reduced positive attitudes towards the self [15]. Studies reporting negative effects were predominantly those manipulating avatar body type, with participants using larger ('obese') bodied avatars reporting lower self-report outcomes or demonstrating a lower performance (conflated with physical activity) in game compared with those in the smaller ("normal") body type conditions. However, it is important to note that none of the studies reporting negative effects had an adequate control condition. Only one study [7] measured attitudes, intentions, and behaviours.

\footnotetext{
${ }^{4}$ Note that the effect size for study [13] was taken from a meta-analysis published by one of the original paper's authors, we were unable to reproduce this effect size
} 


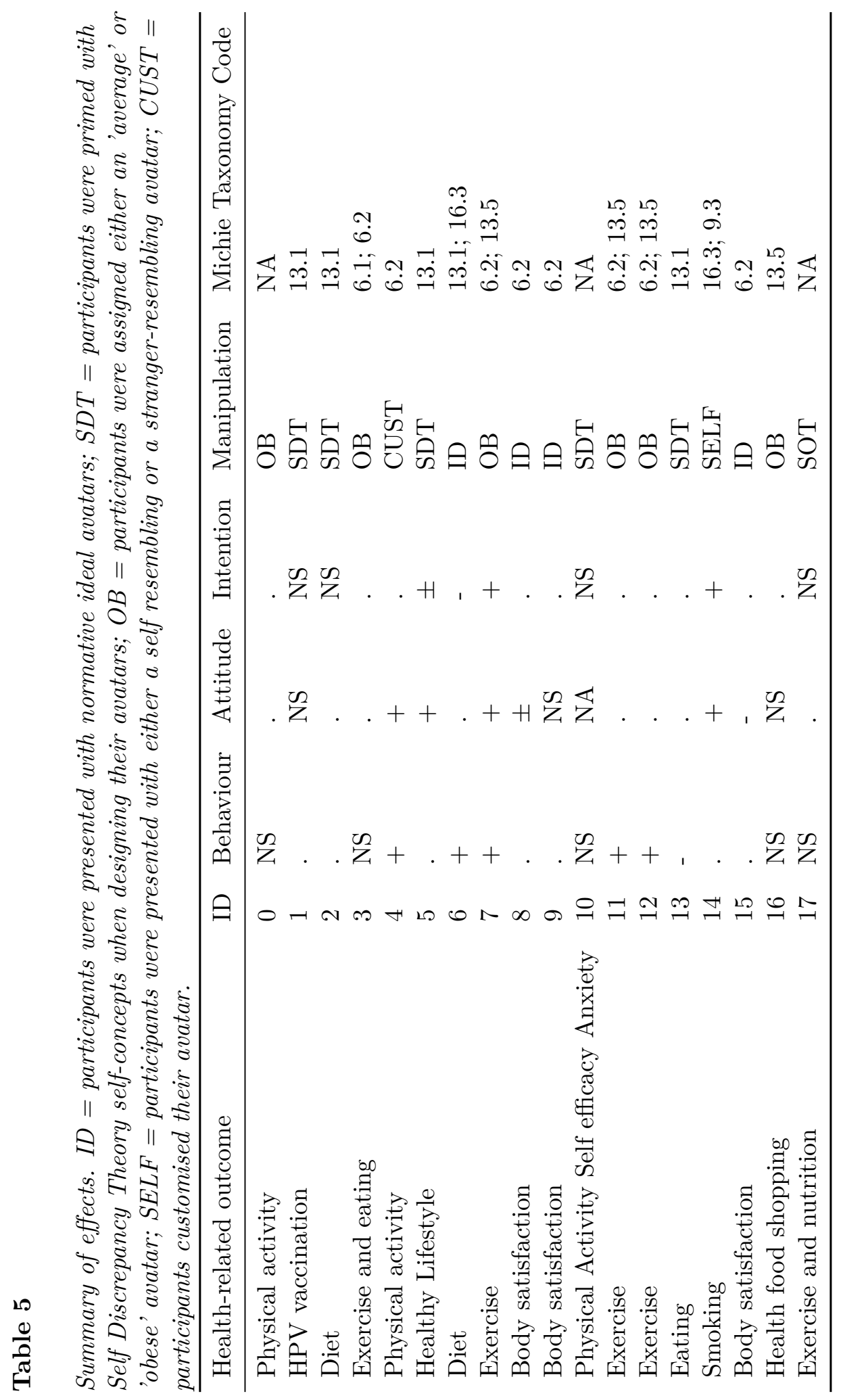


(a) Assigned

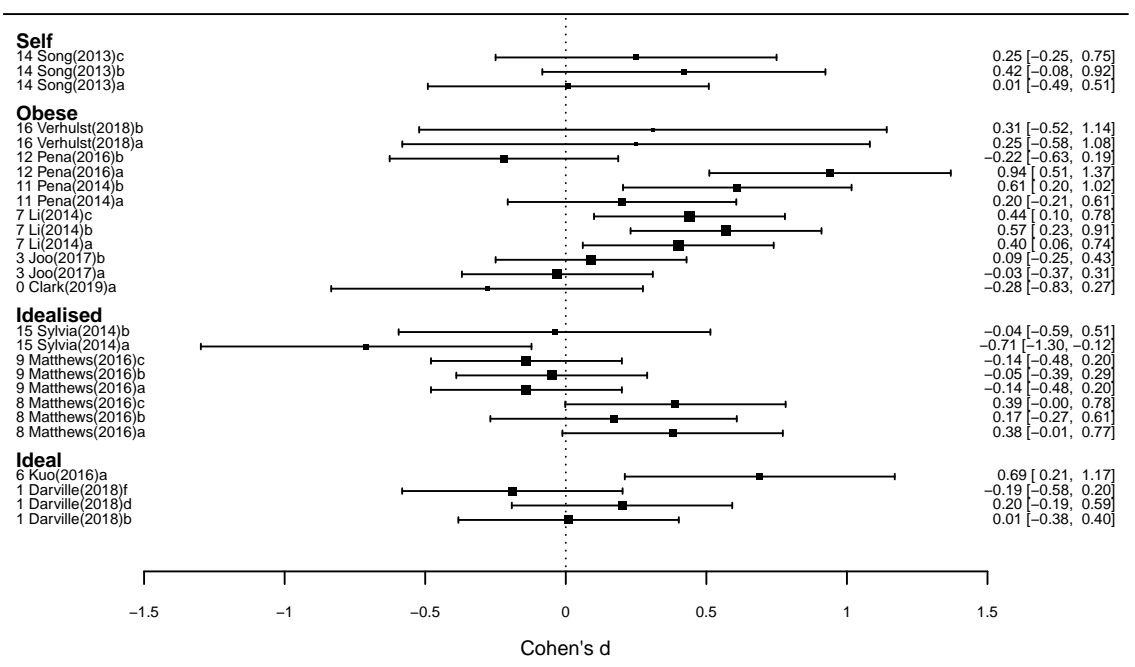

(b) Customised

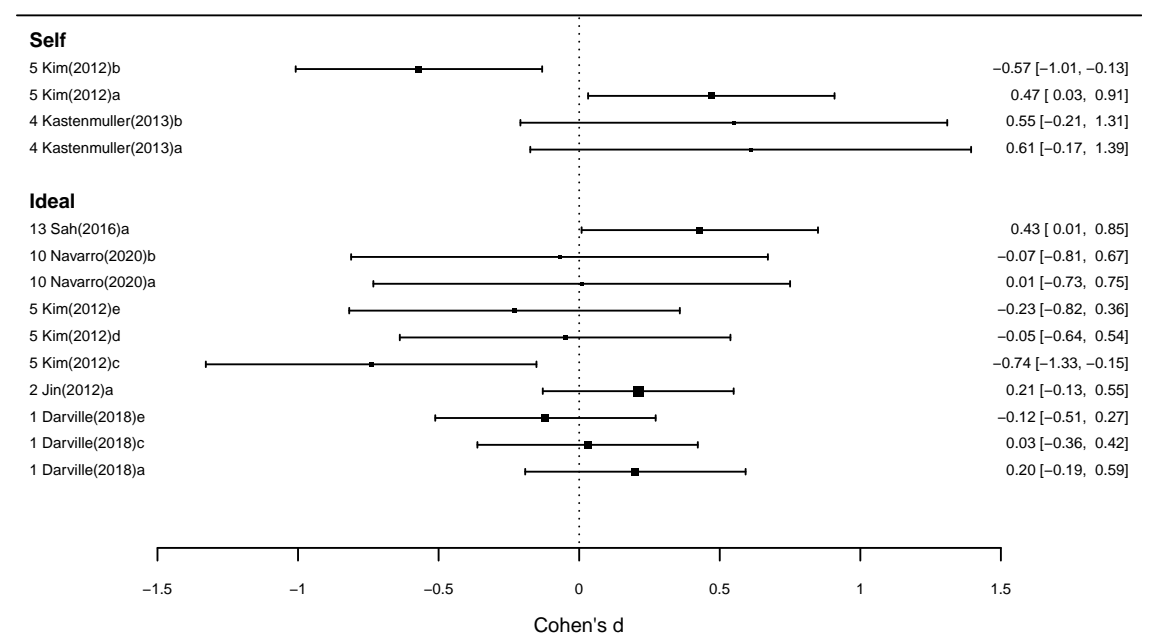

Figure 2

Forest plots of effect sizes for the effect of avatar appearance. Categorised by assignment and customisation

\section{Behaviour.}

Behavioural outcomes included both in-game and post-game measures, and were manipulated using 'Obese' avatars and subjective ideal avatars. In-game dependent variables included the number of healthy virtual food items selected by participants $[12,16]$, and performance of participants in an exergame $[0,7,11,12]$. The aim in study $[13]$ was for participants to practice selecting healthy food with the expectation that this carry-over into real-world food ordering; whereas in [16] there was no apparent real world application - the study merely aimed to demonstrate the Proteus Effect in food selection behaviours. 
(a) Behaviour

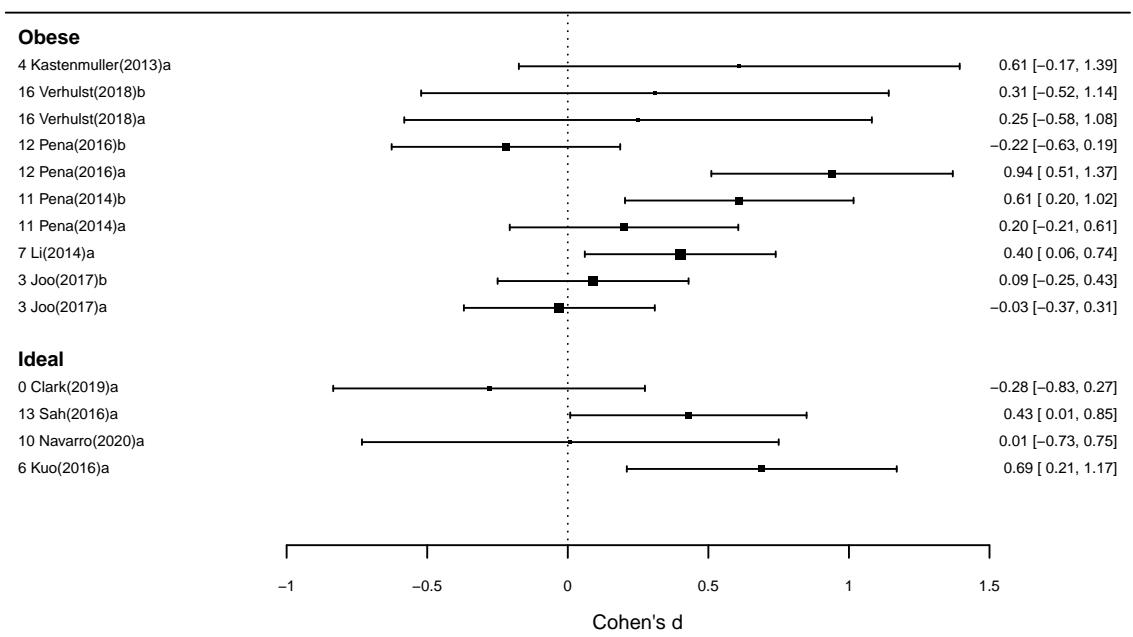

(b) Intentions

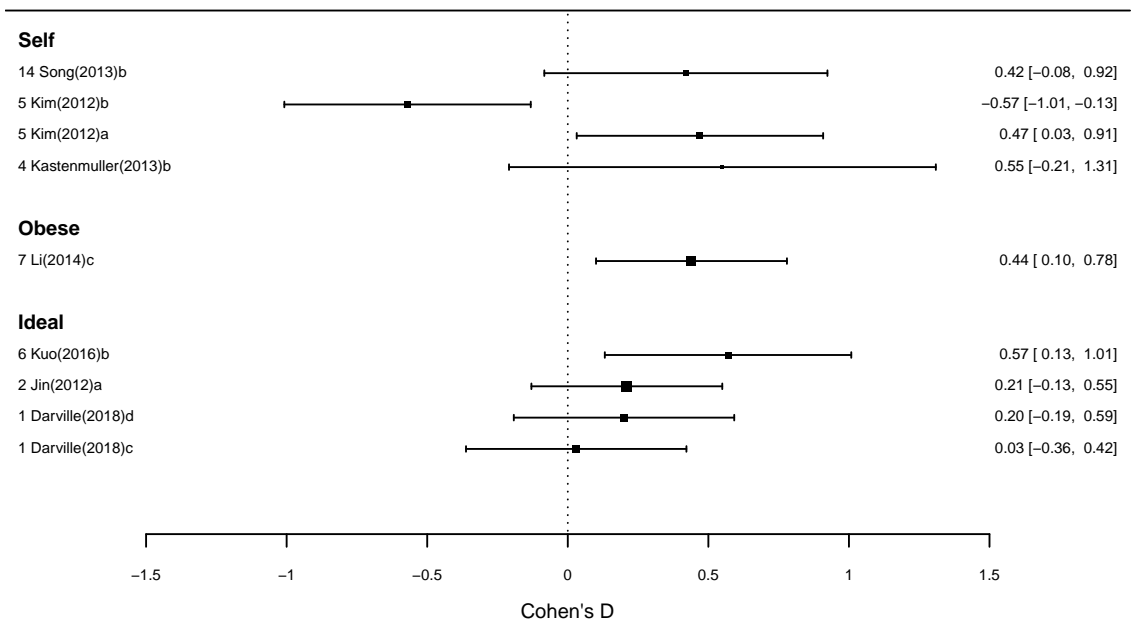

(c) Attitudes and Beliefs

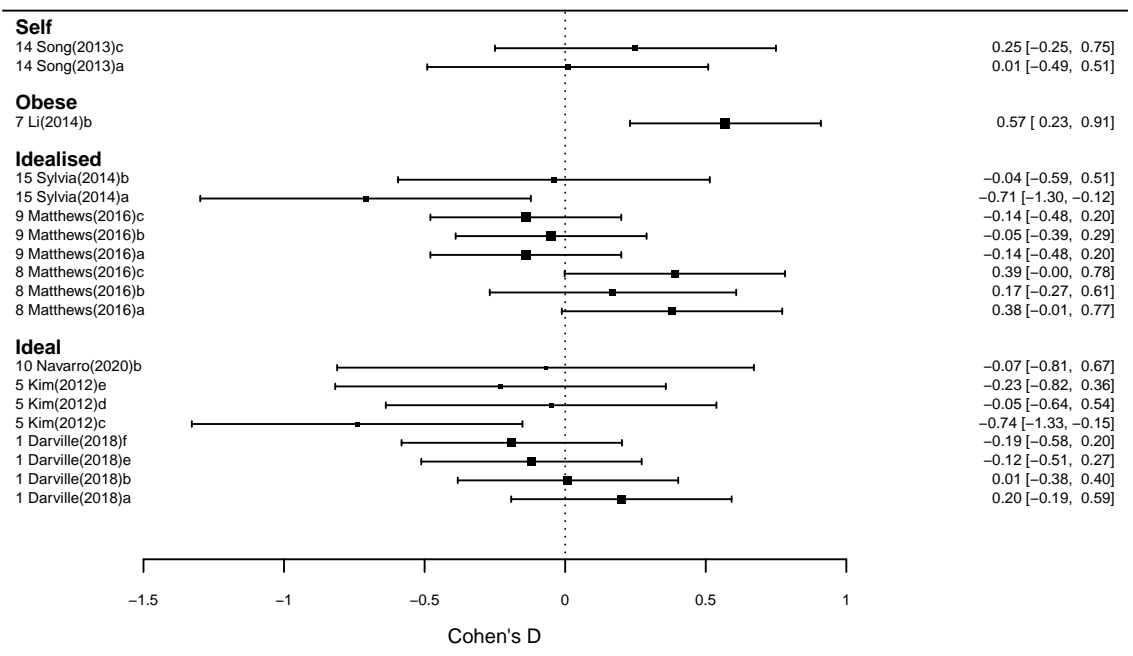

\section{Figure 3}

Forest plots of effect sizes for the effect of avatar appearance. Categorised by manipulation: Categorised by manipulation: Ideal = ideal vs actual self activation, Customised = Customised vs assigned avatar, Obese = larger bodied vs slimmer avatar. 
The degree of in-game performance was the dependent variable in four studies and was measured using accelerometers on the wrist and waist [11,12], and through exergame completion times $[0,7]$, which were used as proxies for exertion. Some studies assessed post-play behaviours, which have different implications. Rather than directly affecting behaviours, the implication is that there is a more complex psycho-social process, such as that predicted by the Theory of Planned Behaviour (Ajzen, 1991), in which the avatar increases self efficacy or behavioural intentions, leading to a greater probability that they will perform a behaviour. Post-test outcomes included number of days a physical activity goal was met in the week between intervention and follow-up [10], the number of steps taken on a stepping machine [3], the quantity of high-sugar food consumed [3,6], and the number of healthy snacks selected by participants [12]. No studies measured long term behaviour change (i.e. $>1$ week).

Effect sizes for slim versus obese avatars on behaviours were mostly positive, meaning that people with slimmer avatars exerted themselves more or made healthier food choices. There were three non-significant exceptions [0a, 3a, 12b] in which participants in the obese avatar condition were more active than those in the slim condition. Two studies demonstrated that health-related behaviours were more likely to be performed when an ideal-self avatar was presented, compared with an actual-self avatar ${ }^{5}$.

\section{Intentions.}

Intentions were measured using self-report methods that were either developed for the study or adapted from previous research. The development of these self-report measures was not discussed in detail, nor was a framework referenced for their development (e.g. Ajzen, 2002). Motivation to exercise was measured in study [10], and a standardised, theory driven framework was used to measure this. Two studies $[5,6]$ attempted direct measures of behavioural intentions using either a coupon selection analogy [5] in which participants were invited to select vouchers for products or services that were either healthy or unhealthy; or through the amount of sugar to be included in a bubble tea reward drink [6]. Study [5] reports the outcome as 'perceived risk to self', however the items appeared to be asking participants the extent to which they intend to perform health-related and risk reducing activities (jogging, visits to a doctor, p1360), and so this was categorised as an intention measure. The development and standardisation of these methods was not discussed within the papers, and so the extent of their validity cannot be determined.

Effect sizes for avatar appearance on avatar appearance were mostly positive across all manipulations, although only four $95 \%$ confidence intervals excluded zero [5a, 5b, 6b, 7c]. However, study [5] reported that people who customised their avatars selected more unhealthy coupons than those who were assigned avatars. This effect was calculated from the scores reported in the authors thesis (Kim, 2010, p. p57).

\section{Attitudes and Beliefs.}

Attitudes and Beliefs were collapsed into one category since the outcomes are conceptualised as proportional to one another (Equation 1 Ajzen, 1991, p. p191), and few

\footnotetext{
${ }^{5} \mathrm{NB}$ study [6] utilised a weight-reduction paradigm, however participants were selected based on their intention to lose weight, and so the assumption that a weight reduced avatar represents a subjective ideal is justified.
} 
studies measured either. Attitudes were measured using a mixture of novel and standardised methods. Although study [4] reported measuring motivation to be physically active, upon inspection the measure seemed lacked face validity and appeared to actually measure attitudes towards jogging. One other study measured attitudes towards health behaviours using questions about participants opinions of exercise [7]. Study [10] included measures of Anxiety towards exercise, Demotivation, and Enjoyment of exercise. Participants' selfreported attitudes towards their bodies were measured in three studies $[8,9,15]$, and included standardised measures such as the Swansea Muscularity Attitudes Questionnaire, Body Esteem Scale and Body Perceptions Scale. Three studies measured perceptions of risk or susceptibility [1,5,14], each of which used novel or adapted measures, and may be interpreted as a belief about the probability that an event (e.g. contracting HPV) may occur.

Overall, the effect of avatar appearance on Attitudes and Beliefs was the weakest of the three outcomes. The majority of the effects were close to zero, or included zero in the 95\% confidence interval. The studies involving idealised avatars appeared to have the largest effect on attitudes, although this seemed dependent on gender, since in studies involving men, attitudes towards their body were reduced, whereas they were increased (albeit nonsignificantly) in women. Five effect sizes were negative [1e,1f,10b,15a,15b], suggesting that provision of some avatars may reduce positive attitudes or beliefs about health-related behaviours or the body, however the $95 \%$ confidence intervals of only two effect did not contain zero.

\section{Use of Health Psychological Theory.}

Table 5 also includes a column indicating which (if any, or the closest match) of the interventions catalogued in the Behaviour Change Technique Taxonomy (BCT) were used in each study (Michie et al., 2013) ${ }^{6}$. This taxonomy was the result of a review of 124 behaviour change techniques followed by several rounds of delphic discussions. These BCTs were reduced down to 93, clustered into 16 groups, each of which have an index number (e.g. 4.3 is the third BCT in cluster 4. The most common 'type' of BCT was Social Comparison (6.2), featuring in seven of the studies (although in some cases attitudes rather than behaviour were the measured outcome). This is to be expected, since avatars provide a visual stimulus for the player, and various theories predict that players will compare the avatar with themselves. Alternative approaches included 'Identifying the Self as a Role Model' (13.1, N =4) and 'Identity Associated with Changed Behaviour' (13.5, $\mathrm{N}=4$ ), both of which were approached from a Self Discrepancy Theory perspective. The supplemental materials from Michie et al. (2009; "Supplemental Material for Effective Techniques in Healthy Eating and Physical Activity Interventions," 2009) also have some relevant categories. For instance, parallels may be drawn between category T9 (Model/demostrate behaviour) [3], T19 (provide opportunities for social comparison) $[0,3,7,8,9,11,12,15,16]$, and T21 (Identification as rolemodel/advocate).

\footnotetext{
${ }^{6}$ This was not included in the pre-registration, and was added after some very useful feedback from a senior colleague.
} 


\section{Assessment of Methodological Risk of Bias}

Assessment of the risk of bias is presented in Table $7^{7}$. The clearest message is that not enough steps were taken to control for (or report) bias in these studies. Two very recent studies provided details about the random allocation method and blinding of study personnel $[0,10]$. Cursory mentions were made by other studies, but in general, there was no indication that steps were taken to blind participants or researchers to assignments or manipulations. Three studies $[7,8,14]$ indicated that participants were allocated prior to arrival, meaning that selection bias could potentially be higher. One study described the method of randomisation as being a permutated block randomisation [5], but it was unclear whether steps were taken to ensure personnel were sufficiently blinded to the allocation. Three studies (two from the same author) included an awareness check to detect participants who had guessed the purpose of the study [5,11,12]. Attrition bias was not suitably addressed, with the majority of studies having discrepancies between the reported sample size and the degrees of freedom reported with their inferential statistics - and in two cases (from the same article) [8,9], the degrees of freedom varied between outcomes within the same study. The majority of studies used self report measures, so observer bias was not applicable. Most studies reported all outcome variables, however in two, [9,11] covariates were omitted from the analysis due to the variable not being a significant predictor, and the values were not reported.

Six statistically significant effects from four studies were categorised as unknown risk of bias. Although this does not mean the studies are biased per se, replications of these effects should be conducted with adequate experimental controls. There were five effects from two studies yielding statistically significant effects in the higher risk of bias category, again this does not mean that the effects should be discarded, but care should be taken when interpreting the results, and authors are urged to implement bias reduction techniques such as blinded assignment and randomisation, awareness checks, and adequate reporting of results, or at least improve the reporting of their use in methods sections. It may be telling that none of the low risk of bias studies yielded statistically significant effects, but the length of the confidence intervals in each case offer the alternative explanation of inadequate sampling; specifying a smallest effect size of interest, testing for equivalence, and conducting a priori power analyses would have allowed further exploration of the nature of these null results.

\footnotetext{
${ }^{7}$ Justifications for these decisions are provided in the supplementary materials
} 
Table 6

\begin{tabular}{llll}
\hline ID & \multicolumn{1}{c}{ Reference } & Adequate sequence generation & Allocation concealment \\
\hline 0 & Clark et al (2019); UK; Randomised Trial & + & + \\
1 & Darville et al (2018) & $?$ & $?$ \\
2 & Jin (2012) & $?$ & $?$ \\
3 & Joo and Kim (2017) & $?$ & $?$ \\
4 & Kastenmüller et al & $?$ & $?$ \\
5 & Kim and Sundar (2012) & $?$ & $?$ \\
6 & Kuo et al (2016) & $?$ & - \\
7 & Li et al (2013) & $?$ & $?$ \\
8 & Matthews et al (2016a) & $?$ & $?$ \\
9 & Matthews et al (2016b) & $?$ & $?$ \\
10 & Navarro et al (2020) & + & + \\
11 & Peña and Kim (2014) & $?$ & $?$ \\
12 & Peña et al (2016) & $?$ & $?$ \\
13 & Sah et al (2016) & $?$ & $?$ \\
14 & Song et al (2013) & $?$ & $?$ \\
15 & Sylvia et al (2014) & $?$ & $?$ \\
16 & Velhurst et al (2018) & $?$ & $?$ \\
17 & Waddell et al (2015) & $?$ & $?$ \\
\hline
\end{tabular}




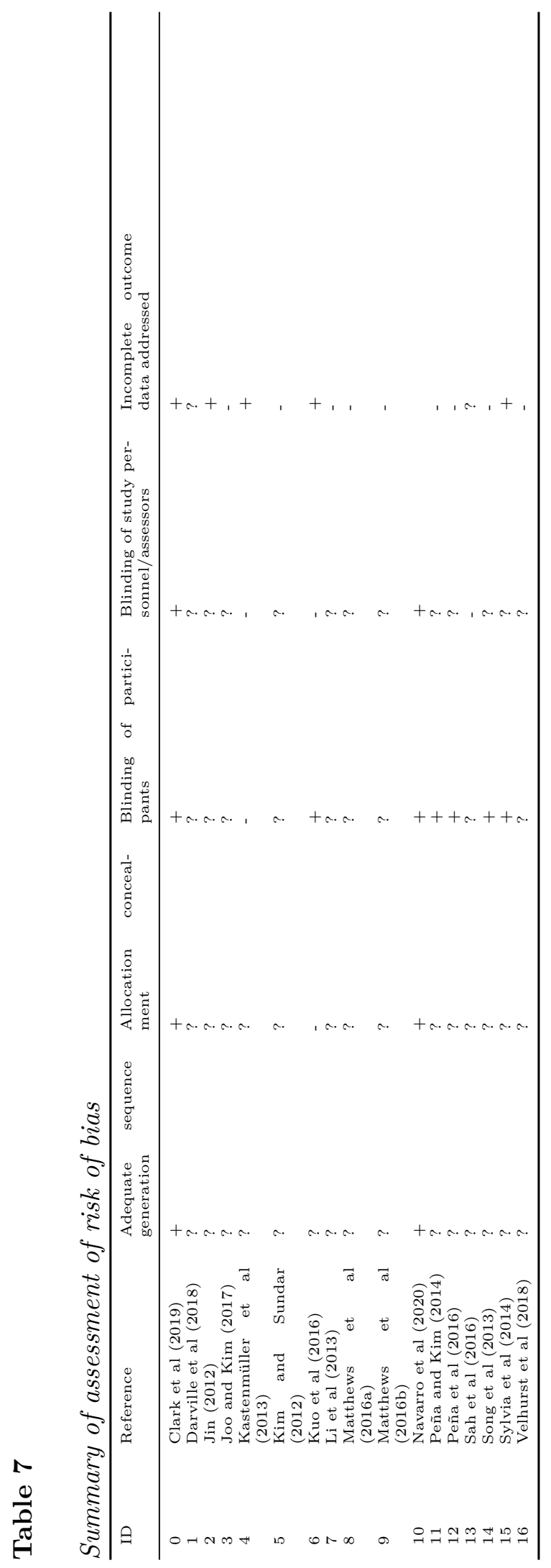




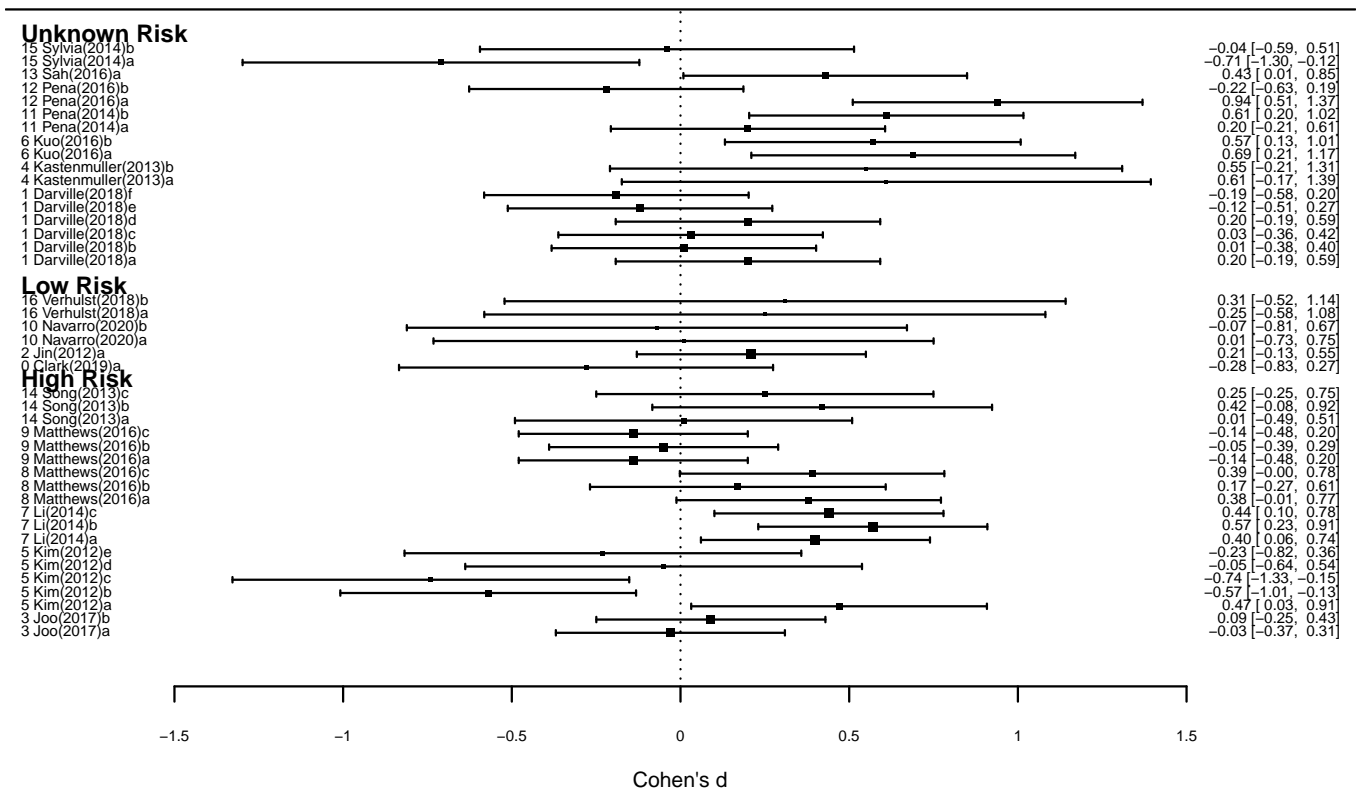

Figure 4

Forest plots of effect sizes for the effect of avatar appearance. Categorised by risk of bias

\section{Sources of Heterogeneity}

There was considerable variation in how the studies were run, how the avatars were chosen or created, the activities that participants performed with the avatar, and the methods used to measure the outcomes. This was evident even after studies were categorised (e.g. by manipulation: ideal, obese, etc). In nine studies, the avatars were from consumer videogames $[2,3,4,7,8,9,11,12,15]$; in one study the $3 \mathrm{D}$ virtual world, SecondLife, was used for the customisation and presentation of avatars [5]; in six studies bespoke or specialist avatar creation software and environments were used $[0,1,6,10,13,14,16]$. In two studies, participants' photographs were used to create the avatar $[6,14]$.

Studies using standardised measures reported fewer significant effects of avatar appearance manipulation $[\mathrm{N}=1]$ than those using modified or bespoke measures $(\mathrm{N}=5)$ (see Figure 5).

Further, the inclusion of covariates was inconsistent throughout, with eight studies reporting their use (two were removed for not being significant). Covariates included weekly game use, gender, BMI, body discrepancy, and body shape concern, and time since last meal. There was no mention of covariates in the hypotheses of the studies, so it is not clear whether these were a priori decisions.

The platform of the study was a further source of heterogeneity. The majority of studies were conducted using desktop computers or consumer consoles, but two used fully immersive virtual reality equipment $[6,16]$. In six of the studies, participants were playing 
exergames $[0,2,4,7,11,12]$, meaning that as they moved, the avatar moved with them. In the remaining studies, participants controlled the avatars with either a console controller or keyboard and mouse.

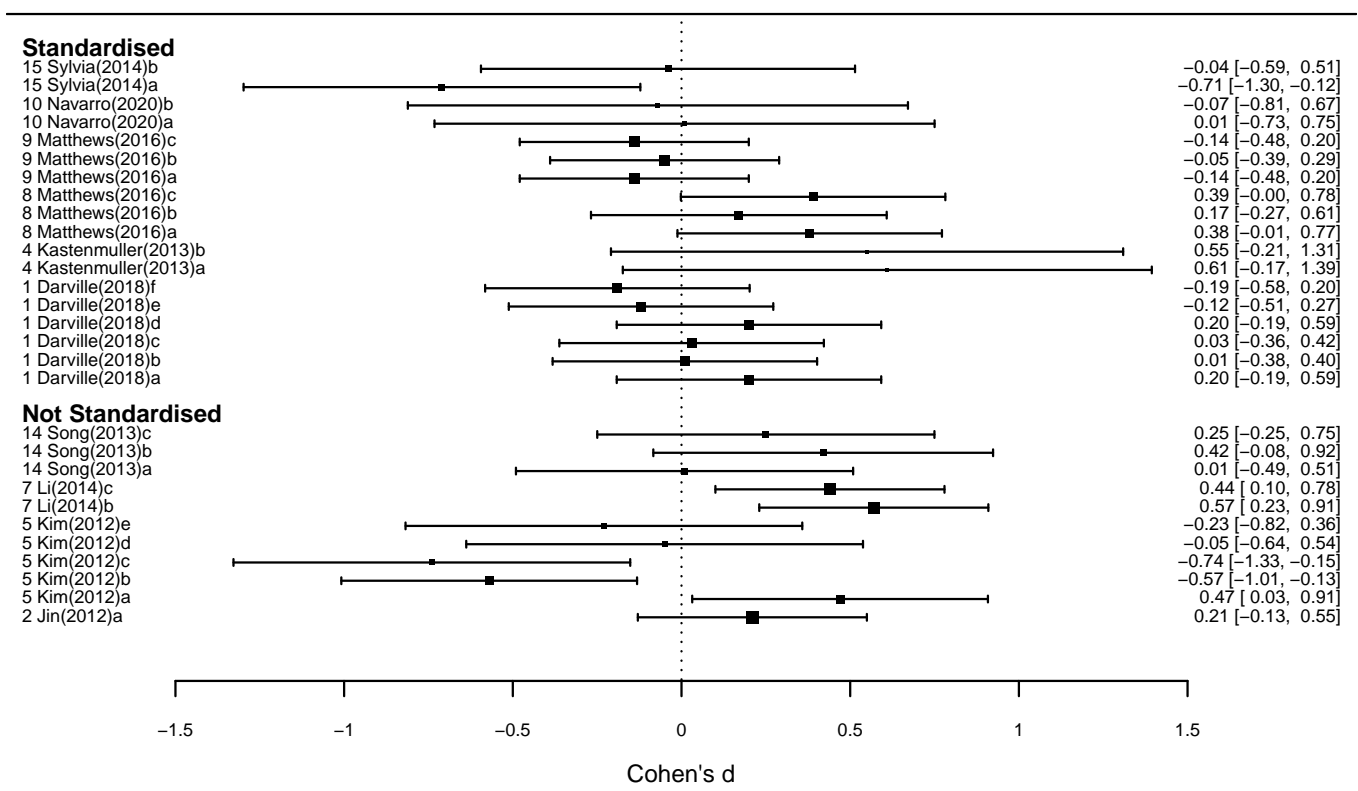

\section{Figure 5}

Forest plots of effect sizes for the effect of avatar appearance. Categorised by risk of bias

\section{Discussion}

The purpose of this review was to assess the evidence that avatar appearance may influence health-related attitudes and behaviours. The results from this review imply a number of potential pathways for the influence of avatars on health-related outcomes, with varying degrees of efficacy. A direct route involves manipulating the appearance of an avatar and altering the user's self-perception. This results in the adoption of behaviours or attitudes that are congruent with the stereotypical traits of the avatar. This route, named the Proteus Effect (Yee et al., 2009), was adopted in five studies [0,7,11,12,16], and weakly adopted in one study [3]. These studies used avatars that were designed to appear either athletic or "obese" /"overweight, with the prediction that these would lead the user to feel that they were fitter (or more"lazy") than they currently were. According to the Proteus Effect, this would result in a temporary increase (or reduction) in exercise exertion during an exergaming session, or selection healthier (or unhealthier) items in food selection tasks. The long term effects of this pathway are understudied in the health literature, and there is only weak evidence that there are long term effects in the general cyberpsychology literature (e.g. Yee, 2007, Pilot Study 4). Study [3] of this review could be seen as an attempt to address post-play behaviour. However, the platform used was The Sims, in which users control created characters using menu instructions, and have no direct control, 
which is arguably not the embodied experience required by the Proteus Effect (hence, weak adoption). The effect sizes for the direct effect of avatar appearance on behaviour were the strongest and most reliable of the studies in the dataset, and as such this pathway could potentially be effective for increasing exertion during play, rather than motivating people to start playing, or eliciting future health-related behaviours. However, the logical conclusion is to not allow people to create or have access to avatars with larger bodies, which feeds into the wider debate about the stigmatisation of weight. Instead of continuing to demonstrate that people are slower when they think they're fat, a more fruitful course may be to explore ways in which this effect may be reduced through positive depictions of larger bodied individuals (Clark et al., n.d.; Pearl et al., 2015).

An indirect route involved allowing players to customise their avatars to represent their actual or ideal selves in game (Higgins, 1996). It is assumed by the authors of these studies that the latter offers an outlet for wish-fulfilment, and that the presentation of such an avatar may reduce the perceived discrepancy between the actual-self, and ideal-self. This ideal self may be athletic and encourage the user to engage in more healthy lifestyles (Kim \& Sundar, 2012). This approach was adopted in six studies $[1,2,5,6,10,13]$ with mixed results. The majority of ideal-self activations had positive effects and a discussion of this incongruence is presented later.

A third, more passive route is related to traditional media theory through Social Comparison Theory (Festinger, 1954). In this, the mere exposure to ideal- and hyperidealised forms may be sufficient to induce social comparisons, and reduce a person's body satisfaction, and by association exercise intentions or behaviours (Crawford \& Eklund, 1994; Hall et al., 2011; Homan et al., 2012; Wasilenko et al., 2007). By this line of reasoning, using an athletic, idealised, or hyper-idealised avatar would result in upward social comparisons, and negative feelings towards the self. However, this simple interpretation has been criticised for suggesting that audiences are passive, critically unengaged with media content, and evidence in favour is limited (Ferguson, 2013; Gill, 2012). The passive approach was adopted in three studies $[8,9,15]$. The predicted effects were non-significant in $[8,9]$, and effect sizes suggest that men are affected negatively by exposure to idealised avatars, whereas women are (non-significantly) positively affected. Clearly, replications are needed to confirm this. If mere exposure to idealised avatars is sufficient to induce body dissatisfaction, then dissatisfaction ought to occur in studies involving athletic-looking opponents, such as those used in the two studies by Pena et al $[11,12]$, and future studies investigating the benefits of athletic over fat avatars might benefit from including body esteem measures.

\section{Implications for Wider Health Behaviour Literature}

There was a notable absence of health-psychology theory in many of the studies; with most favouring social psychological (e.g. SDT or the Proteus Effect) theories applied to health. The most common health-psychology theory to be cited was the Theory of Planned Behaviour (Ajzen, 1991), and this was mostly for the behavioural intention construct $[1,2,4,5,6,7,14]$, although attitudes and beliefs also featured. Other constructs within the Theory of Planned Behaviour, such as perceived social norms, and perceived behavioural

control were absent and would have been useful inclusions. One study [1] used elements 
of both the Health Belief Model (Becker, 1974) and the Theory of Planned Behaviour to make predictions, and used self-efficacy (Bandura, 2001), a broad analogue for perceived behavioural control in the Theory of Planned Behaviour. No studies reported using the recommended methods for designing TPB-based tools, e.g. (Ajzen, 2002).

Although none of the studies in this review can strictly be regarded as interventions, they may be approximately positioned within the wider taxonomy of health-related behaviour interventions (Michie et al., 2009, 2013). Michie et al's Identity associated with changed behavior was the closest to the Proteus Effect, although this would actually predict the reverse: Behaviour associated with changed identity. This could represent a distinct category that could be added to a future version of the taxonomy. Only one study utilised avatars to apply a perceived Punishment in the form of time acceleration [14]. It might be advantageous for HCI researchers to cast a wider net around existing entries to the BCT taxonomy, and for Health Psychology researchers to consider including the contributions of $\mathrm{HCI}$ research in future versions of the BCT and alternatives.

\section{Evidence of Effectiveness}

In addition to table 5 which provides an indication as to whether the effect of interest was significant or not, Figures ?? and ?? provide forest plots of the effect size and confidence interval estimate for each effect in the study. The following sections discuss the effects in each of the two main cateogories (Assigned and Customised avatars).

\section{Assigned Avatars.}

The most consistently successful paradigm was the manipulation of avatar body size to affect in-game performance. However, performance was the outcome in only four studies, with the remainder of 'obese avatar' studies testing post-game behaviours [3] or virtual shopping decisions [16]. Two of the studies used identical methods (virtual tennis game) on college students of different genders [11,12]. The third [7] was conducted on a cohort of larger bodied children, and utilised a running game on the Nintendo Wii. Study [0] replicated the method of study [7] but on adults who were not selected based on body size, and reported (non-significantly) lower mean track completion times for participants in the larger bodied condition than the athletic condition. To say that the effect is reliable would be premature, and substantial work is required to confirm this.

In the two studies by Peña et al [11,12], people were selected based on being in the "normal" Body Mass Index (BMI) range. The intention appears to have been for the participants to perform downwards comparisons on the larger sized avatar, and lateral or upwards comparisons on the smaller avatar. Participants in study [0] were not screened for body size. In the study by $\mathrm{Li}$ et al [7], participants were selected based on being in the higher BMI range, and would therefore be expected to perform lateral comparisons on the larger avatars, and upwards comparisons on the smaller avatars. The absence of baseline performance in any of the studies makes it unclear whether the participants were performing or self reporting at, below, or above their true attribute.

Only one study found significant effects of avatar body type on attitudes, intentions and behaviours [7]. In the remaining studies, these measures were either not significant 
[15], or not measured $[0,3,6,11,12]$. Moreover, exposure to normative ideals, and hyper-ideal avatars had small, noisy, or mixed effects on body image related outcomes. For instance, muscularity had no effect on male body satisfaction in study [9], but did on one scale in study [15]; and in study [8], women self reported more favourable attitudes towards their weight, but less favourable body satisfaction. Taken as a whole, it is unclear whether merely controlling an avatar will affect psychosocial constructs, whereas the effect on behaviour during play may warrant further investigation.

\section{Customised Avatars.}

Evidence of the effect of avatar customisation on the outcomes of interest was also mixed, with the direction of effects varying both between and within studies. Three studies directly compared assignment with customisation, but only one [5] reported significant differences in a measures of self-reported intentions a behavioural analogue for intentions was not significant. This is unexpected, since if they were measuring the same construct, the direction of the effect ought to be the same. Future studies on customisation and intentions might include measures of identification (Turkay \& Kinzer, 2014) and self presence (Ratan, 2010) which have been shown to influence motivation to play tradition and exercise videogames (Birk et al., 2016; Li \& Lwin, 2016)

The type of instructions given to participants customising their avatars was manipulated in five studies $[1,2,5,10,13]$. The instructions were based on self-concepts and models described in Self Discprepancy Theory (SDT; Higgins, 1987), namely actual (perception of the self as it currently is), ideal (projection of who a person desires to be), or ought selves (projection of who a person feels they should morally be). Mere customisation based on these concepts had null $[1,2,10]$, positive [13] and negative influences [5] on health-related outcomes. The actual self was used as an avatar creation prime in Study [5], with the hypothesis that it would lead to higher perceived risk to the body. An effect was found in one of the three scales, and the reason for choosing the significant scale appeared to be data-driven. Study [1] also used actual-self activation but found no significant differences between the actual and ideal avatars on any outcome (perceived risk, self efficacy, behavioural intentions). Other studies used actual self activation as a baseline [2,13], with ideal and ought self avatars forming the experimental condition. There were no significant differences reported between groups on any health related outcomes in studies comparing actual and ideal selves $[1,2,5,10,13]$. The ought self model was used in one study [13], and the authors reported that when controlling for health consciousness, participants selected fewer food items compared with those in the actual body-activation condition. What the authors did not discuss is that when not controlling for health consciousness, and when all participants were analysed together, the ought self had a stronger, and significant negative effect on the observed quantity of healthy foods eaten. Finally, none of these studies actually measured self discrepancy to see if the avatar exposure had actually worked in the intended way.

\section{Quality of evidence}

No study met the stringent reporting standards of clinical trials set out by the

Cochrane Tool for Assessing Risk of Bias (Higgins et al., 2011). All significant effects were 
found in studies with either high or unknown risk of bias. This is not to say the studies was biased, but there was insufficient information provided to make judgements on many of the CTAB bias domains, and ultimately this means that no firm conclusions may be made about how the studies were run. This is likely to be an issue with the field in general, which is only just starting to adopt reporting standards. However, Navarro et al. (2020, p. 10) were a notable exception, who made a substantial effort to debias their study. They implemented randomisation and blinding methods to safeguard their study. This study as also the most recent in the set, and it is hoped that future authors will follow their lead.

In three studies, participants were asked to report on the nature of the study, and those who guessed the purpose were excluded from further analysis. However, in all but two studies $[0,10]$, no information about the blinding of conditions or allocation concealment was provided, with only a cursory mention being made to the randomisation of participants. Two studies mentioned that allocation was performed prior to the participants arriving, which could indicate a higher risk of selection bias.

No studies reported conducting an a priori power analysis to inform their sample size, nor was a smallest effect size of interest (SEOI) declared, with the majority of studies conflating significance with relevance. Power was only mentioned by Jin [2], who reported an a posteriori 'observed power' estimate after the sample had been collected and the effect size estimate observed. It has been convincingly argued that observed power provides little information of use by meta-scientists and statisticians, because the observed effect may be inflated due to outliers having a disproportionate influence on the overall estimate (Baguley, 2004).

The validity of measures used within the studies was not discussed in any of the articles. This is important because few of the studies directly measured behaviours, with most relying on the estimation of psychosocial constructs. Without a discussion of the construct validation of these measures it is impossible to judge whether the items are appropriate for the purpose (Cronbach \& Meehl, 1955; Flake \& Fried, 2019).

One area in which risk of bias was high was attrition. The degrees of freedom reported by the majority of the studies $(\mathrm{N}=14)$ do not match the reported sample size, suggesting that the analyses were run on different samples, with no discussion of attrition. Although these differences may be due to typos, Statcheck was run on all inferential statistics and no errors were detected (Epskamp \& Nuijten, 2015).

\section{Sources of heterogeneity}

There was considerable variation in the uses of avatars to affect health-related outcomes. A potential source of variation includes the level of interaction with the avatar. In nine of the experiments, participants had no control over the appearance of the avatar, and so any self similarity would be coincidental. In the remaining three studies, participants selected [1], or customised $[1,2,4,12]$ that looked like themselves. All customisation instructions involved some level of self similarity, that is participants in these experiments were limited in their freedom to self-present. 
The choice of dependent variable was also a potentially large source of heterogeneity. These ranged from self-report measures to direct observation; from post-treatment, to peri-treatment behaviours. The domain of the dependent variable did not always match the treatment. For example, in one case [2], the treatment was an exercise game and the outcome was healthy eating intentions. An argument could be made for the higher success of studies at finding an effect when there was a close, theory-driven, correspondence between the dependent variable and the treatment. For example, the Proteus Effect predicts that stereotype-relevant behaviours will be adopted when an avatar visually represents this group. If this were to follow into the muscularity studies, the Proteus Effect would predict variations of in-game behaviours, rather than post-play attitudes towards the self.

A further source of heterogeneity was the reason for conducting the study, and the implicative chain that led to the hypotheses of the studies. The Proteus Effect was the primary avatar theory in nine studies $[0,2,3,5,7,11,12,13,16]$, whereas Virtual Self Modelling was in one study [14]. Four did not cite any of the main avatar-influence theories $[1,6,8,9]$. In addition to these avatar influence frameworks, there was a large variety of ancillary theories attached to the chains of many of the studies. Self Discrepancy Theory (Higgins, 1987) $[1,2,5,6,13]$ and Social Comparison Theory (Festinger, 1954) $[5,8,9,11,12]$ were cited in five, Social Cognitive Theory (Bandura, 2001) was cited in four studies [2,3,5,11], and Stereotype Threat (Steele \& Aronson, 1995) [7] and Regulatory Fit (Higgins, 1996) [2] were used as second factors in one study.

There were more significant effects of avatar appearance on non-standardised measures than standardised measures. This is not presented as evidence of questionable research and/or measurement practices, but future authors are urged to pre-register their items, and follow the guidance provided by Flake and Fried (2019) when reporting and developing measurement items.

\section{General Discussion}

Perhaps the most consistent result from the body of work reviewed here is that playing an exercise game as an "obese" avatar makes players move less than if they were playing with smaller avatars. Three studies, conducted on different populations, reported statistically significant results on this effect. There have been no published attempts to replicate these findings, although one unpublished study [0] attempted to replicate and extend these findings. It would be possible to explore an inverted U-shaped relationship between avatar appearance and exercise behaviour, predicting optimal behavioural influence around a person's ideal body type, and decreasing as a function of the absolute distance from that ideal. This would require a great deal of further work.

The models used in studies $[0,11,12]$ were lean and muscular, and likely did not reflect an 'average' (i.e. non-idealised) body type. The avatars in study [7] appeared very thin. Indeed, the definition of "normal" amongst studies is ill-defined, and a baseline would need to be established prior to further work being conducted. Further, many of the studies were conducted in countries with very different cultural norms to one another. There were studies conducted in the UK, Japan, Singapore, Taiwan, and the USA. This may represent a further source of heterogeneity, since the experience of participants between these studies 
may be different in relation to culturally-determined body ideals (Grogan, 2017, pp. pp31$35)$.

Two studies [6,14] investigated the effect of the 'future self'. These used manipulated images as avatars, with study [6] reducing the weight of the avatar to the participants ideal, and study [14] adding smoking-related age artefacts to the face. These studies yielded fair results and there has been some compelling research on appearance related interventions for a range of health behaviours including UV protection (Persson, Grogan, et al., 2018; Persson, Benn, et al., 2018) and smoking (Grogan et al., 2011), as well as applications in non-avatar based virtual reality studies (Ahn, 2015a). As such, this may be a fruitful direction for future research.

\section{Grounding in Theory}

In moving forward with avatar-related health interventions, it is perhaps more important to ensure that what is already known is incorporated into future research than it is to continue demonstrating novel examples of the same effects. The theories that have been used to develop the studies in this review make very specific predictions, and these need to be taken into account when progressing the field.

\section{Proteus Effect.}

In general terms, the mechanisms behind behavioural assimilation from avatars are described by the Proteus Effect (Yee, 2007; Yee et al., 2009). Under this theory, embodying an avatar results in the user drawing inferences from the avatar's appearance, and behaving in a congruent way. It is not sufficient to simply state that changing an avatar's appearance will result in health behaviour change, since the Proteus Effect has a number of necessary conditions that must be met for behaviour to change. To wit, there must be a visually activated stereotype (positive or negative) associated with the target behaviour; the avatar must unambiguously represent this stereotype; the stereotype must be general enough that participants will have encountered it. Only four studies in the set met these conditions $[0,7,11,12]$, and three of these found significant, and relatively strong effects results of ingame performance. There is a wide literature on weight stigma, and a persistently evident belief that larger individuals are lazy, slow, and lack control (Flint \& Reale, 2018; Seacat \& Mickelson, 2009). It is perhaps unsurprising then that the manipulation of avatars to have larger, stigmatised body-types would affect behaviour. However, since this effect is reliant on social constructs (stereotypes), it should not be regarded as a medication for larger bodied people, as suggested by Li et al. (2014) (p45), since stereotypes are subject to change and may be manipulated experimentally and sociologically. Further, the stereotype that fat people are lazy is inherently negative and stigmatic, but currently has the benefit of being deemed socially acceptable by various power structures (Gillborn et al., 2020). It is inconceivable that other stigmatised views (e.g. related to race, gender) would be prescribed in the $21^{\wedge}\{$ st $\}$ century; and instead work ought to be focused at reducing the weight-related Proteus Effect, rather than maintaining it.

As of yet there is no published literature on stereotypes related to idealised and hyper-idealised body types. An unpublished account suggests that these stereotypes may 
be ambivalent, eliciting both positive (determination) and negative (arrogant, cold) activations (Clark, 2019, Chapters 6,7,\& 8), both of which may influence in game behaviour. and the studies that attempted to manipulate body satisfaction using avatars relied on traditional media influence theories. Such studies have been shown to be weak, and only have an effect in very specific circumstances (e.g. women with pre-existing body image dissatisfaction, Ferguson, 2013). In the current review, it appeared that men's body satisfaction was more affected by hyper-idealised avatars than women. The absence of a literature on stereotypes, and the observation that body satisfaction might be reduced under exposure to idealised avatars suggests that the negative consequences are better established than the potential positive consequences. Absent literature on stereotypes related to muscularity, predictions cannot be made under the Proteus Effect - this step needs to be taken before broad recommendations about the avatars that people should or should not have access to are made.

\section{Self-Discrepancy.}

Similar arguments to those presented above may be directed at the avatar customisation studies. In general, the studies aimed to promote health related behaviours using self concepts from Self Discrepancy Theory. The chain of reasoning in Kim and Sundar's study [5], is that when people create an ideal avatar, they observe their distorted self perceptions from the third person perspective, which results in reflexive self perceptions, carrying over into their behaviour as per the predictions of Self Perception Theory (Bem, 1972). This chain of reasoning is not clearly supported by the literature. Higgins (1987) states that Self Discrepancy Theory also has necessary conditions for the activation of a discrepancy. A particular self concept must $a$. exist, and $b$. be accessible. At no point in any of the customisation studies was the presence of self discrepancy in participants established. SelfDiscrepancy measurement typically has an ideographic and nomothetic component, that is, the participant states their ideal, ought, or feared attributes and then indicates the distance their actual self is from these attributes (Hardin \& Lakin, 2009). The simple inclusion of such a measure would allow researchers to predict the effect of avatar influence on behaviour more precisely. Participants were provided with instructions to create avatars that represented their actual, ideal, or ought selves, without determining whether such discrepancies existed, or what the nature of the discrepancy was. The assumption within the studies was that participants would make a healthy version of themselves, but participants only had access to visual features. There is a body of work suggesting that the ideals of some individuals are rooted in functionality rather than appearance (Body Appreciation, Tylka \& Wood-Barcalow, 2015). The ideal image of people who self-rate highly on Body Appreciation may look identical to how they currently look, but (for instance) be able to do a backflip, or hold a plank for eight hours. Without determining whether a person has health behaviour internalised, it is not possible to know what the avatar actually represents to them. In Kim and Sundar's study [5], participants were simply asked to "customise their avatar to resemble their [actual/ideal] self" - and so then if there is an underlying avatar persuasion mechanism, it is unclear what is being activated when the avatar is used, and which behaviours mapped onto the representations. 


\section{Comparison with Previous Reviews}

A side-by-side comparison between the two previous systematic reviews is presented in Table 8. There are key differences in the interpretation of the results, with the two previous reviews being more positive about the effect of avatars on health-related behaviours, whereas the current review presents a more critical view of the field.

There are several similarities between the Rheu et al. (2019) review and the current review. Both attempted to synthesise evidence on the extent to which avatars may affect health-related behaviours; with a particular focus on exercise and dieting. However, this article benefits from being pre-registered, with transparent decisions and justifications throughout. Moreover, the discussion of the studies is focused on a single attribute of avatars: their appearance. The definition of an avatar-based intervention in the current review was one in which the avatar was the main manipulation. This led to the exclusion of studies such as Behm-Morawitz et al. (2016) and Christensen et al. (2013), in which the environment was the primary difference between conditions. As mentioned earlier, the findings from Rheu et al. (2019) may be inflated by the inclusion of studies that used doppelgangers rather than avatars (e.g. Fox \& Bailenson, 2009; Ruiz et al., 2012), and the exclusion of unpublished, non-significant studies from doctoral theses (e.g. Fox, 2010).

Several of the studies captured by the Horne et al. (2019) review were part of the full-text review procedure of the current review, but were excluded on the basis that they did not manipulate an element of the avatar's appearance as an independent variable (e.g. Behm-Morawitz et al., 2016; Napolitano et al., 2013). The Horne review was also more restrictive in its target population, only looking at "obese" and "overweight" populations.

Unlike the current review, strength of evidence in both previous reviews was predicated solely on $p$ values rather than an interpretation of effect sizes and confidence intervals, allowing for a more nuanced description of the effects. 


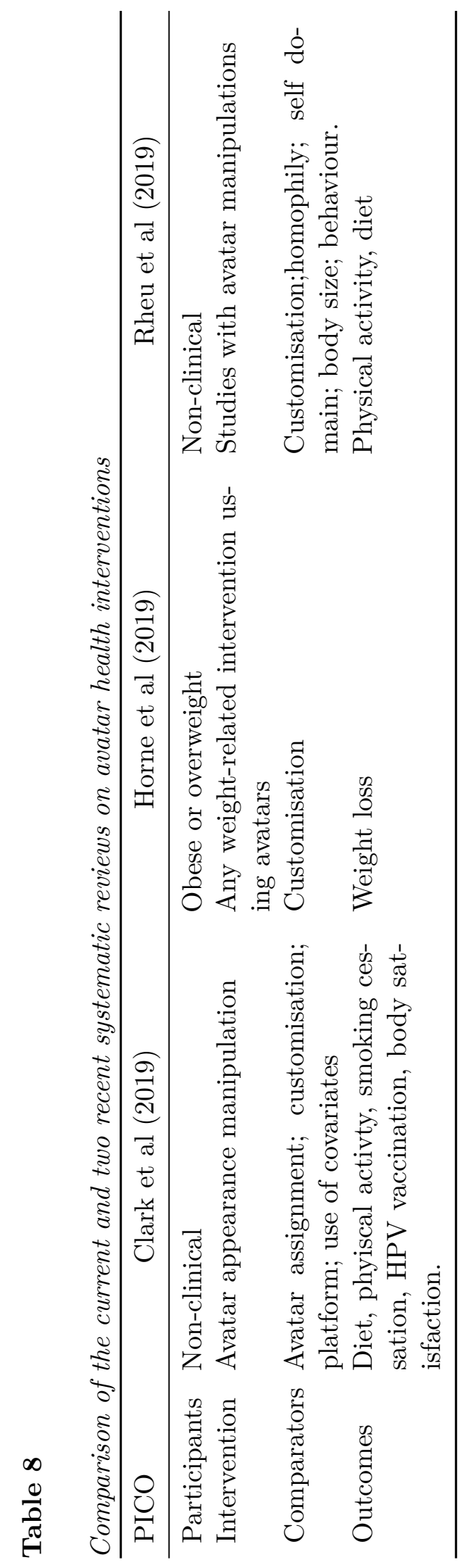




\section{Strengths/Limitations}

Although there have been at least four qualitative reviews on avatars and health behaviour (Ahn, 2015b; Ahn \& Fox, 2017a; Horne et al., 2019; Rheu et al., 2019), this study is the first to systematically collect and assess the evidence avatar appearance on health-related attitudes, beliefs, intentions, and behaviours. The previous reviews present preliminary findings on how doppelgängers, as well as avatars, can influence health behaviours, as well as how these may be integrated with social psychology theories to produce favourable health-related outcomes. The current review assessed studies investigating the use of only avatar-appearance to influence health behaviours, presented effect size estimates and confidence intervals, and addressed methodological and theoretical flaws. It is hoped that it will be used in conjunction with the existing reviews for future avatar researchers to plan their studies.

A strength of the study was the use of an established risk of bias tool. However, the Cochrane Risk of Bias tool is designed for clinical trials, and much of the information that would be provided to support low bias (e.g. randomisation protocols and measures to reduce allocation concealment) is not commonly included in psychology and human factors research. The effect sizes were also plotted against the risk of bias, suggesting that outcomes may have been influenced by biased methods.

One important finding was that there are surprisingly few studies on this topic, with a large and varied array of effect comparisons. This also meant that the estimation of a meta-analytic effect size would not have been informative.

It is important to note that, although various authors were contacted regarding filedrawer studies, and non-peer reviewed sources were searched (e.g. ProQuest thesis repository), only one unpublished study was included, and this was produced by the research team who wrote the current review. One study had previously been a Masters thesis. This is a limitation because given the near ubiquity of publication bias in Psychology (Simonsohn et al., 2014), and in videogame violence research (Ferguson, 2007), it is likely that this review does not contain all studies conducted studies this field. Further, the inclusion criteria for the studies had to be adapted, since the pre-registered criteria were at once too broad and too narrow. That is, they stated that any study using an avatar would be included, and that outcomes had to be measured before and after the intervention.

\section{Conclusion}

This review reaches a different conclusion to that of Rheu et al. (2019). Where the previously published review suggests that when an avatar is "fit, customisable, and demonstrating health consequences" (p9), health-related behaviours may be improved; and that self-discrepancy related constructs could be used to benefit users health-related behaviours, we conclude that there is as yet insufficient evidence to support the use of avatar appearance as health-related interventions, although there are many opportunities to improve the area. We note that the effect sizes for customised avatars were generally weak, and many included zero in the $95 \%$ confidence interval. We also conclude that where the depiction of larger bodies may influence health-reated behaviours, this should not be seen as a remedy, but as 
a bias that needs to be remedied. We argue that people need to be motivated to exercise independently of body shape, and focus on function and fitness rather than shape. Further, the potential consequences of limiting avatar body sizes to athletic or "normal" ranges on body satisfaction need to be explored. More theoretical work is needed to place avatars within the wider taxonomy of health-related behaviour change; the predictions made by existing theory need to be revisited; and replication attempts are required to explicate the interaction between avatars and real world health-related behaviour. 


\section{References}

References marked with an asterisk indicate studies included in the systematic review.

Ahn, S. J. (2015a). Incorporating immersive virtual environments in health promotion campaigns: A construal level theory approach. Health Communication, $30(6), 545-556$.

Ahn, S. J. (2015b). Using avatars and agents to promote real-world health behavior changes. The Digital Patient: Advancing Healthcare, Research, and Education, $171-180$.

Ahn, S. J., \& Fox, J. (2017a). Immersive virtual environments, avatars, and agents for health.

Ahn, S. J., \& Fox, J. (2017b). Virtual environments, avatars, and agents in health and risk messaging. In Oxford Research Encyclopedia of Communication. Ed. Oxford University Press. https://doi.org/10.1093/acrefore/9780190228613.013. 325

Ahn, S. J., Fox, J., \& Hahm, J. M. (2014). Using virtual doppelgängers to increase personal relevance of health risk communication. International Conference on Intelligent Virtual Agents, 1-12.

Ahn, S. J., Johnsen, K., Robertson, T., Moore, J., Brown, S., Marable, A., \& Basu, A. (2015). Using virtual pets to promote physical activity in children: An application of the youth physical activity promotion model. Journal of Health Communication, 20(7), 807-815.

Ajzen, I. (1991). The theory of planned behavior. Organizational Behavior and Human Decision Processes, 50(2), 179-211.

Ajzen, I. (2002). Constructing a tpb questionnaire: Conceptual and methodological considerations.

Aust, F., \& Barth, M. (2017). papaja: Create APA manuscripts with R Markdown. https://github.com/crsh/papaja

Baguley, T. (2004). Understanding statistical power in the context of applied research. Applied Ergonomics, 35(2), 73-80.

Bandura, A. (2001). Social cognitive theory: An agentic perspective. Annual Review of Psychology, 52(1), 1-26.

Becker, M. H. (1974). The health belief model and personal health behavior. Health Education Monographs, 2, 324-473.

Behm-Morawitz, E., Lewallen, J., \& Choi, G. (2016). A second chance at health: How a $3 D$ virtual world can improve health self-efficacy for weight loss management among adults. 19(2), 74-79. https://doi.org/10.1089/cyber.2015.0317

Bem, D. J. (1972). Self-perception theory. Advances in Experimental Social Psychology, 6, 1-62. 
Birk, M., Atkins, C., Bowey, J., \& Mandryk, R. (2016). Fostering intrinsic motivation through avatar identification in digital games. 2982-2995.

Christensen, J. L., Miller, L. C., Appleby, P. R., Corsbie-Massay, C., Godoy, C. G., Marsella, S. C., \& Read, S. J. (2013). Reducing shame in a game that predicts hiv risk reduction for young adult men who have sex with men: A randomized trial delivered nationally over the web. Journal of the International AIDS Society, $16(3)$.

* Clark, O. J. (2019). On the persuasive power of videogame avatars on health-related behavioir [PhD thesis]. Manchester Metropolitan University.

Clark, O. J., Grogan, S., Cole, \& Jennifer, M. (n.d.). "A sort of half-way house, a hero version of me": Men and women's accounts of creating their ideal-self avatar and being portrayed as normative ideal in an exercise videogame.

Crawford, S., \& Eklund, R. C. (1994). Social physique anxiety, reasons for exercise, and attitudes toward exercise settings. Journal of Sport and Exercise Psychology, $16(1), 70-82$.

Cronbach, L. J., \& Meehl, P. E. (1955). Construct validity in psychological tests. Psychological Bulletin, 52(4), 281.

* Darville, G., Anderson -Lewis, C., Stellefson, M., Lee, Y.-H., MacInnes, J., Pigg, R. M., Gilbert, J. E., \& Thomas, S. (2018). Customization of avatars in a hpv digital gaming intervention for college-age males: An experimental study. Simulation \& Gaming, 49(5), 515-537. https://doi.org/10.1177/1046878118799472

Del Re, A. (2013). Compute. Es: Compute effect sizes. $R$ package version 0.2-2. R-project.

Elmagarmid, A., Fedorowicz, Z., Hammady, H., Ilyas, I., Khabsa, M., \& Ouzzani, M. (2014). Rayyan: A systematic reviews web app for exploring and filtering searches for eligible studies for cochrane reviews. Evidence-Informed Public Health: Opportunities and Challenges, 9.

Epskamp, S., \& Nuijten, M. (2015). Statcheck: Extract statistics from articles and recompute $p$ values (r package version 1.0. 1.).

Ferguson, C. J. (2007). Evidence for publication bias in video game violence effects literature: A meta-analytic review. Aggression and Violent Behavior, 12(4), $470-482$.

Ferguson, C. J. (2013). In the eye of the beholder: Thin-ideal media affects some, but not most, viewers in a meta-analytic review of body dissatisfaction in women and men. Psychology of Popular Media Culture, 2(1), 20.

Festinger, L. (1954). A theory of social comparison processes. Human Relations, $7(2), 117-140$.

Flake, J. K., \& Fried, E. I. (2019). Measurement schmeasurement: Questionable measurement practices and how to avoid them. 
Flint, S. W., \& Reale, S. (2018). Weight stigma in frequent exercisers: Overt, demeaning and condescending. Journal of Health Psychology, 23(5), 710-719.

Fox, J. (2010). The use of virtual self models to promote self-efficacy and physical activity performance [PhD thesis]. Stanford University.

Fox, J., \& Bailenson, J. N. (2009). Virtual self-modeling: The effects of vicarious reinforcement and identification on exercise behaviors. Media Psychology, 12(1), $1-25$.

Freijy, T., \& Kothe, E. J. (2013). Dissonance-based interventions for health behaviour change: A systematic review. British Journal of Health Psychology, $18(2), 310-337$.

Gill, R. (2012). Media, empowerment and the "sexualization of culture" debates. Sex Roles, 66(11), 736-745. https://doi.org/10.1007/s11199-011-0107-1

Gillborn, S., Rickett, B., Muskett, T., \& Woolhouse, M. (2020). Apocalyptic public health: Exploring discourses of fatness in childhood 'obesity'policy. Journal of Education Policy, 35(1), 3-22.

Grogan, S. (2017). Body image: Understanding body dissatisfaction in men, women and children. Taylor \& Francis.

Grogan, S., Flett, K., Clark-Carter, D., Conner, M., Davey, R., Richardson, D., \& Rajaratnam, G. (2011). A randomized controlled trial of an appearance-related smoking intervention. Health Psychology, 30(6), 805.

Hall, E. E., Baird, S. A., Gilbert, D. N., Miller, P. C., \& Bixby, W. R. (2011). Viewing television shows containing ideal and neutral body images while exercising: Does type of body image content influence exercise performance and body image in women? Journal of Health Psychology, 16(6), 938-946. https://doi.org/10.1177/1359105310396394

Hardin, E. E., \& Lakin, J. L. (2009). The integrated self-discrepancy index: A reliable and valid measure of self-discrepancies. Journal of Personality Assessment, $91(3), 245-253$.

Higgins, E. T. (1987). Self-discrepancy: A theory relating self and affect. Psychological Review, 94 (3), 319.

Higgins, E. T. (1996). The" self digest": Self-knowledge serving self-regulatory functions. Journal of Personality and Social Psychology, 71(6), 1062.

Higgins, J. P. T., Altman, D. G., Gøtzsche, P. C., Jüni, P., Moher, D., Oxman, A. D., Savović, J., Schulz, K. F., Weeks, L., \& Sterne, J. A. C. (2011). The cochrane collaboration's tool for assessing risk of bias in randomised trials. BMJ, 343. https://doi.org/10.1136/bmj.d5928

Homan, K., McHugh, E., Wells, D., Watson, C., \& King, C. (2012). The effect of viewing ultra-fit images on college women's body dissatisfaction. Body Image, 9(1), 50-56. https://doi.org/https://doi.org/10.1016/j.bodyim.2011.07.006 
* Horne, M., Hill, A., Murells, T., Ugail, H., Chinnadorai, R., Hardy, M., \& others. (2019). Using avatars in weight management settings: A systematic review. Internet Interventions, 100295.

* Jin, S.-A. A. (2012). Self-discrepancy and regulatory fit in avatar-based exergames. Psychological Reports, $111(3), 697-710$.

* Joo, Y. K., \& Kim, K. (2017). When you exercise your avatar in a virtual game: The role of avatars' body shape and behavior in users' health behavior. Interacting with Computers, 29(3), 455-466.

* Kastenmüller, A., Greitemeyer, T., Fairclough, S., Waite, D., \& Fischer, P. (2013). Playing exergames and sporting activity. Social Psychology.

Khabsa, M., Elmagarmid, A., Ilyas, I., Hammady, H., \& Ouzzani, M. (2015). Learning to identify relevant studies for systematic reviews using random forest and external information. Machine Learning, 1-18. https://doi.org/10.1007/s10994$015-5535-7$

Kim, Y. (2010). CAN your avatar improve your health? THE impact of avatar customization.

* Kim, Y., \& Sundar, S. S. (2012). Visualizing ideal self vs. Actual self through avatars: Impact on preventive health outcomes. Computers in Human Behavior, 28 (4), 1356-1364. https://doi.org/http://dx.doi.org/10.1016/j.chb.2012.02.021

* Kuo, H.-C., Lee, C.-C., \& Chiou, W.-B. (2016). The power of the virtual ideal self in weight control: Weight-reduced avatars can enhance the tendency to delay gratification and regulate dietary practices. CyberPsychology, Behavior \&5 Social Networking, 19(2), 80-85. http://search.ebscohost.com/login.aspx?direct= true $\& \mathrm{db}=$ buh\&AN $=113040553 \&$ site $=$ ehost-live

Li, B. J., \& Lwin, M. O. (2016). Player see, player do testing an exergame motivation model based on the influence of the self avatar. Computers in Human Behavior, 59, 350-357. https://doi.org/http://dx.doi.org/10.1016/j.chb.2016.02.034

* Li, B. J., Lwin, M. O., \& Jung, Y. (2014). Wii, myself, and size: The influence of proteus effect and stereotype threat on overweight children's exercise motivation and behavior in exergames. GAMES FOR HEALTH: Research, Development, and Clinical Applications, 3(1), 40-48.

Matallaoui, A., Koivisto, J., Hamari, J., \& Zarnekow, R. (2017). How effective is "exergamification"? A systematic review on the effectiveness of gamification features in exergames. Proceedings of the 50th Hawaii International Conference on System Sciences.

* Matthews, N. L., Lynch, T., \& Martins, N. (2016). Real ideal: Investigating how ideal and hyper-ideal video game bodies affect men and women. Computers in Human Behavior, 59, 155-164. https://doi.org/https://doi.org/10.1016/j.chb. 2016.01.026 
McCallum, S. (2012). Gamification and serious games for personalized health. PHealth, 85-96.

Michie, S., Abraham, C., Whittington, C., McAteer, J., \& Gupta, S. (2009). Effective techniques in healthy eating and physical activity interventions: A meta-regression. Health Psychology, 28(6), 690701. http://search.ebscohost.com.mmu.idm.oclc.org/login.aspx?direct=true\& $\mathrm{db}=$ pdh\&AN $=2009-20990-006 \&$ site $=$ ehost-live

Michie, S., Richardson, M., Johnston, M., Abraham, C., Francis, J., Hardeman, W., Eccles, M. P., Cane, J., \& Wood, C. E. (2013). The behavior change technique taxonomy (v1) of 93 hierarchically clustered techniques: Building an international consensus for the reporting of behavior change interventions. Annals of Behavioral Medicine, 46(1), 81-95. https://doi.org/10.1007/s12160-013-9486-6

Moher, D., Shamseer, L., Clarke, M., Ghersi, D., Liberati, A., Petticrew, M., Shekelle, P., \& Stewart, L. A. (2015). Preferred reporting items for systematic review and meta-analysis protocols (prisma-p) 2015 statement. Systematic Reviews, 4(1), 1.

Morgan, A. Z., Ulrich, P., Simmons, K. P., Gropper, S. S., Connell, L. J., Daniels, M. K., Latham, E., \& Keiley, M. K. (2014). Effectiveness of a multi-faceted, school-based health intervention program with 4 th graders in alabama. Children and Youth Services Review, 37, 46-54.

Napolitano, M. A., Hayes, S., Russo, G., Muresu, D., Giordano, A., \& Foster, G. D. (2013). Using avatars to model weight loss behaviors: Participant attitudes and technology development. Journal of Diabetes Science and Technology, 7(4), $1057-1065$.

* Navarro, J., Cebolla, A., Llorens, R., Borrego, A., \& Baños, R. M. (2020). Manipulating self-avatar body dimensions in virtual worlds to complement an internetdelivered intervention to increase physical activity in overweight women. International Journal of Environmental Research and Public Health, 17(11), 4045.

Nowak, K. L., \& Fox, J. (2018). Avatars and computer-mediated communication: A review of the definitions, uses, and effects of digital representations. Review of Communication Research, 6, 30-53.

Orji, R., Mandryk, R. L., Vassileva, J., \& Gerling, K. M. (2013). Tailoring persuasive health games to gamer type. Proceedings of the Sigchi Conference on Human Factors in Computing Systems, 2467-2476.

Pearl, R. L., Dovidio, J., \& Puhl, R. M. (2015). Visual portrayals of obesity in health media: Promoting exercise without perpetuating weight bias. Health Education Research, 30(4), 580-590.

* Peña, J., Khan, S., \& Alexopoulos, C. (2016). I am what i see: How avatar and opponent agent body size affects physical activity among men playing exergames. Journal of Computer-Mediated Communication, 21(3), 195-209. https://doi. org/10.1111/jcc4.12151 
* Peña, J., \& Kim, E. (2014). Increasing exergame physical activity through self and opponent avatar appearance. Computers in Human Behavior, 41, 262-267.

Persson, S., Benn, Y., Dhingra, K., Clark-Carter, D., Owen, A. L., \& Grogan, S. (2018). Appearance-based interventions to reduce uv exposure: A systematic review. British Journal of Health Psychology, 23(2), 334-351.

Persson, S., Grogan, S., Dhingra, K., \& Benn, Y. (2018). "It's bit of an eye opener"a qualitative study of women's attitudes towards tanning, sun protection and a facial morphing intervention. Psychology \&6 Health, 33(3), 381-397.

Ratan, R. (2010). Self-presence, explicated. 60th Annual Conference of the International Communication Association, Singapore.

* Rheu, M., Jang, Y., \& Peng, W. (2019). Enhancing healthy behaviors through virtual self: A systematic review of health interventions using avatars. Games for Health Journal.

Ruiz, J. G., Andrade, A. D., Anam, R., Aguiar, R., Sun, H., \& Roos, B. A. (2012). Using anthropomorphic avatars resembling sedentary older individuals as models to enhance self-efficacy and adherence to physical activity: Psychophysiological correlates. Studies in Health Technology and Informatics, 173, 405-411.

* Sah, Y. J., Ratan, R., Tsai, H.-Y. S., Peng, W., \& Sarinopoulos, I. (2017). Are you what your avatar eats? Health-behavior effects of avatar-manifested self-concept. Media Psychology, 20(4), 632-657.

Seacat, J. D., \& Mickelson, K. D. (2009). Stereotype threat and the exercise/ dietary health intentions of overweight women. Journal of Health Psychology, 14 (4), 556-567. https://doi.org/10.1177/1359105309103575

Simonsohn, U., Nelson, L. D., \& Simmons, J. P. (2014). P-curve: A key to the file-drawer. Journal of Experimental Psychology: General, 143(2), 534.

Sirriyeh, R., Lawton, R., Gardner, P., \& Armitage, G. (n.d.). Reviewing studies with diverse designs: The development and evaluation of a new tool. Journal of Evaluation in Clinical Practice, 18(4), 746-752. https://doi.org/10.1111/j.13652753.2011.01662.x

* Song, H., Kim, J., Kwon, R. J., \& Jung, Y. (2013). Anti-smoking educational game using avatars as visualized possible selves. Computers in Human Behavior, 29(5), 2029-2036.

Steele, C. M., \& Aronson, J. (1995). Stereotype threat and the intellectual test performance of african americans. Journal of Personality and Social Psychology, $69(5), 797$.

Supplemental material for effective techniques in healthy eating and physical activity interventions: A meta-regression. (2009). Health Psychology. https://doi.org/ 10.1037/a0016136.supp

* Sylvia, Z., King, T. K., \& Morse, B. J. (2014). Virtual ideals: The effect of video game play on male body image. Computers in Human Behavior, 37, 183-188. 
Turkay, S., \& Kinzer, C. K. (2014). The effects of avatar-based customization on player identification. International Journal of Gaming and Computer-Mediated Simulations (IJGCMS), 6(1), 1-25.

Tylka, T. L., \& Wood-Barcalow, N. L. (2015). The body appreciation scale-2: Item refinement and psychometric evaluation. Body Image, 12, 53-67. https: //doi.org/https://doi.org/10.1016/j.bodyim.2014.09.006

Valentine, J. C., Pigott, T. D., \& Rothstein, H. R. (2010). How many studies do you need? A primer on statistical power for meta-analysis. Journal of Educational and Behavioral Statistics, 35(2), 215-247.

* Verhulst, A., Normand, J.-M., Lombart, C., Sugimoto, M., \& Moreau, G. (2018). Influence of being embodied in an obese virtual body on shopping behavior and products perception in vr. Frontiers in Robotics and AI, 5, 1-20.

Viechtbauer, W. (2010). Conducting meta-analyses in R with the metafor package. Journal of Statistical Software, 36(3), 1-48. http://www.jstatsoft.org/v36/i03/

Waddell, T. F., Sundar, S. S., \& Auriemma, J. (2015). Can customizing an avatar motivate exercise intentions and health behaviors among those with low health ideals? Cyberpsychology, Behavior, and Social Networking, 18(11), 687-690.

Wasilenko, K. A., Kulik, J. A., \& Wanic, R. A. (2007). Effects of social comparisons with peers on women's body satisfaction and exercise behavior. International Journal of Eating Disorders, 40(8), 740-745. https://doi.org/10.1002/eat.20433

Yee, N. (2007). The proteus effect: Behavioral modification via transformations of digital self-representation $[\mathrm{PhD}$ thesis]. Stanford University.

Yee, N., Bailenson, J. N., \& Ducheneaut, N. (2009). The proteus effect: Implications of transformed digital self-representation on online and offline behavior. Communication Research, 36(2), 285-312. 